\title{
Systematical study on superconducting radio frequency elliptic cavity shapes applicable to future high energy accelerators and energy recovery linacs
}

\author{
Valery Shemelin, Shahnam Gorgi Zadeh, Johann Heller, and Ursula van Rienen \\ Universität Rostock, Institut für Allgemeine Elektrotechnik, Albert-Einstein-Straße 2, \\ D-18059 Rostock, Germany
}

(Received 25 May 2016; published 25 October 2016)

\begin{abstract}
Elliptic cavities at medium- and high- $\beta$ range are receiving broader use in the particle accelerator applications. Optimizing the shape of these cavities is a complex and demanding process. In this paper we propose an optimization approach to minimize the ratio of peak magnetic field to the acceleration field $H_{\mathrm{pk}} / E_{\mathrm{acc}}$ while keeping the ratio of peak surface electric field to the accelerating field $E_{\mathrm{pk}} / E_{\mathrm{acc}}$, aperture radius and wall slope angle $\alpha$ at some permitted values. We show that it is possible to substantially vary the cavity geometry without violating the constraints or deteriorating the objective of the optimization. This gives us freedom in designing the geometry to overcome problems such as multipactor while maintaining the minimal $H_{\mathrm{pk}} / E_{\mathrm{acc}}$. The optimization is then performed to find a set of optimized geometries with minimum $H_{\mathrm{pk}} / E_{\mathrm{acc}}$ for different $\beta$ 's ranging from 0.4 to 1 , different peak surface electric fields, wall slope angles and aperture radii. These data could be generally used as a suitable starting point in designing elliptic cavities.
\end{abstract}

DOI: 10.1103/PhysRevAccelBeams.19.102002

\section{INTRODUCTION}

As it was stated in [1] the interest in proton accelerators has necessitated the development of structures that bridge the gap between $\beta=1$ cavities for electrons and low- $\beta$ resonators for heavy ions. All the superconducting radiofrequency (SRF) cavities in operation today for the speedof-light particles are of the same design: they are so-called elliptic cavities. Their geometry can be extended to lower $\beta$ by reducing the length of the cells.

The attractive feature of the elliptic cavities is their simple geometry and therefore relative cheapness compared to other types of SRF cavities used for lower $\beta$ [2], like different $\lambda / 4$ or $\lambda / 2$ transmission line cavities or multielement spoke cavities. So, the attempt to expand the area of elliptic cavities to lower $\beta$ 's is natural, and it is realized in many laboratories.

Any superconducting cavity is an expensive structure and it should be properly optimized to obtain maximal acceleration rate $E_{\mathrm{acc}}$. A hard limiting barrier in the SRF cavities is the maximal (or peak) surface magnetic field $H_{\mathrm{pk}}$. So, minimization of the value of $H_{\mathrm{pk}} / E_{\mathrm{acc}}$ is the first task for the cavity optimization. Minimization of this value is closely related to the decrease of $\mathrm{rf}$ losses in the cavity which defines the cost of operation. However, some

\footnotetext{
*shahnam.zadeh@uni-rostock.de
}

Published by the American Physical Society under the terms of the Creative Commons Attribution 3.0 License. Further distribution of this work must maintain attribution to the author $(s)$ and the published article's title, journal citation, and DOI. circumstances make necessary deviations from the optimal shape: higher order modes, multipactor, Lorentz force detuning and others.

In this paper, minimization of the $H_{\mathrm{pk}} / E_{\mathrm{acc}}$ is done for given values of $E_{\mathrm{pk}} / E_{\mathrm{acc}}$, where $E_{\mathrm{pk}}$ is the peak surface electric field. The proposed algorithm is interchangeable, and, conversely, minimization of the $E_{\mathrm{pk}} / E_{\mathrm{acc}}$ can be done for given values of $H_{\mathrm{pk}} / E_{\mathrm{acc}}$ if the field emission is the dominated factor for the operation field gradient. However, we see a little reason to do this work because the peak electric field, or, more precisely, $E_{\mathrm{pk}} / E_{\mathrm{acc}}$, to prevent field emission, is already limited from above as a primary parameter in the present paper.

In this work we try to systematically compare the properties of elliptic cavities at different $\beta$, to understand limitations from the lower values of $\beta$ and to suggest some solutions of the multipactor problem.

We found that some substantial deviations of the shape can be done without deterioration of the value of $H_{\mathrm{pk}} / E_{\mathrm{acc}}$. This property opens the way to overcome above-mentioned problems with multipactor, higher order modes, and Lorentz forces keeping minimal peak magnetic field and cryogenic losses.

\section{ELLIPTIC GEOMETRY AND SURFACE FIELDS}

Optimization of an elliptic cavity consists of the proper choice of geometrical parameters for a contour described by two elliptic arcs and a straight segment tangent to both of them, Fig. 1. 
Three of these parameters, length of the half-cell $L$, the aperture radius $R_{a}$, and the equatorial radius $R_{\text {eq }}$ are not a subject of optimization. They are defined by other physical requirements: $L$ should be equal to the quarter of the wave length, so that the particle could be effectively accelerated in each cell of a multicell cavity, the aperture is defined by requirements to coupling between cells and by the level of wake fields that could be allowed for a given project, the equatorial radius $R_{\mathrm{eq}}$ is used for tuning the cavity for a given frequency.

From the remaining six parameters shown in Fig. 1 ( $A$, $B, a, b, d, \gamma)$, four only are needed to fully describe the geometry. Here $A$ and $B$, also as $a$ and $b$ are the half-axes of the equatorial and iris constitutive ellipses, respectively.

Some authors follow [3] and use combinations of these parameters: $R=B / A$, the equator ellipse aspect ratio and $r=b / a$, the iris ellipse aspect ratio, also as the wall distance from the iris plane $d$ and the wall angle inclination $\gamma$. So, we have four dissimilar primary parameters: $R, r, d$, and $\gamma$.

The reason of this choice is explained [3] as follows: $R$ defines a local minimum of the peak surface magnetic field, $r$ defines a local minimum of the peak surface electric field, $d$ allows to reduce the capacitive volume in favor of the magnetic volume and vice versa, in order to balance the peak surface magnetic and electric fields on the cavity walls, $\gamma$ influences the mechanical behavior of the cavity and controls its inductive volume.

This explanation looks convincing and physically feasible. However, change of the angle $\gamma$ or the permitted value of $E_{\mathrm{pk}} / E_{\mathrm{acc}}$ leads to the necessity to optimize again the values of $R$ and $r$. Besides, there is a dependence of the local minimum of the magnetic field on the iris aspect ratio though not so strong as on the equator aspect ratio, and conversely, the electric peak field minimum depends on the

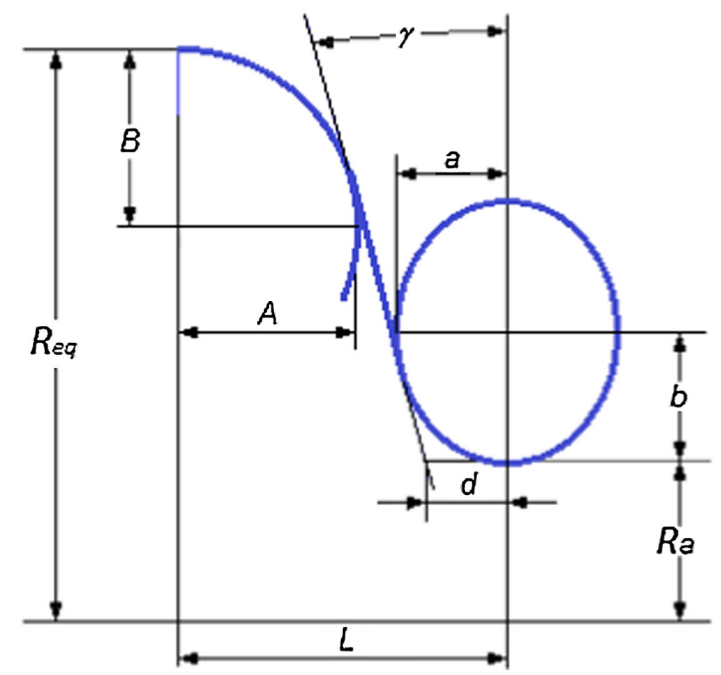

FIG. 1. Cell shape parametrization.

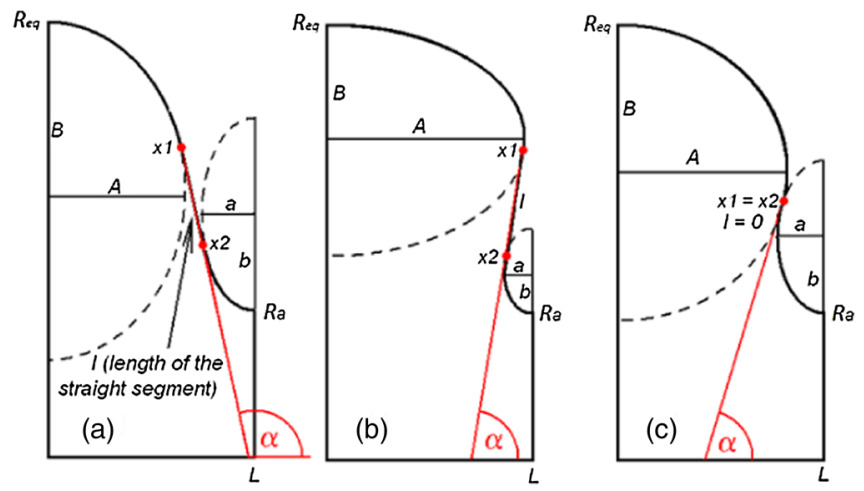

FIG. 2. Geometry of the elliptic half-cell: non-reentrant-(a), and reentrant-(b) and (c). Angle $\alpha=90^{\circ}+\gamma$, compare to Fig. 1.

equator ellipse aspect ratio. On the other hand, the choice of four half-axes lengths $(A, B, a$, and $b)$ as the primary parameters for optimization simplifies the description and does not increase the work for optimization.

Recalculation from $A / B, a / b, d$ and $\gamma$ to $A, B, a$ and $b$ can be easily done solving a system of algebraic equations.

Equations defining half-axes of the smaller ellipse, $a$ and $b$, and also coordinates of the point of conjugation $\left(x_{2}, y_{2}\right)$ (point $x 2$ in Fig. 2), taking as given the values of $\gamma, L, R_{a}, d$ and $r$, are the following:

$$
\begin{array}{r}
\tan \gamma=\frac{L-d-x_{2}}{y_{2}-R_{a}}, \\
\frac{\left(L-x_{2}\right)^{2}}{a^{2}}+\frac{\left(y_{2}-R_{a}-b\right)^{2}}{b^{2}}=1, \\
b\left(L-x_{2}\right) \tan \gamma=a \sqrt{a^{2}-\left(L-x_{2}\right)^{2}}, \\
b / a=r .
\end{array}
$$

Half-axes of the bigger ellipse, $A$ and $B$, and coordinates of the point of conjugation $\left(x_{1}, y_{1}\right)(x 1$ in Fig. 2), taking additionally as given the values of $R_{\mathrm{eq}}$ and $R$, are the following:

$$
\begin{gathered}
\tan \gamma=\frac{x_{2}-x_{1}}{y_{1}-y_{2}}, \\
\frac{x_{1}^{2}}{A^{2}}+\frac{\left(y_{1}-R_{\mathrm{eq}}+B\right)^{2}}{B^{2}}=1, \\
B x_{1} \tan \gamma=A \sqrt{A^{2}-x_{1}^{2}}, \\
B / A=R .
\end{gathered}
$$

We will use this set of primary geometric parameters for optimization ( $A, B, a$, and $b$ ), as well as it is used in many other projects [4-6]. Some details of the geometry used in the future discussion are shown in Fig. 2. We will measure the angle of the wall inclination as the angle $\alpha$ between the 

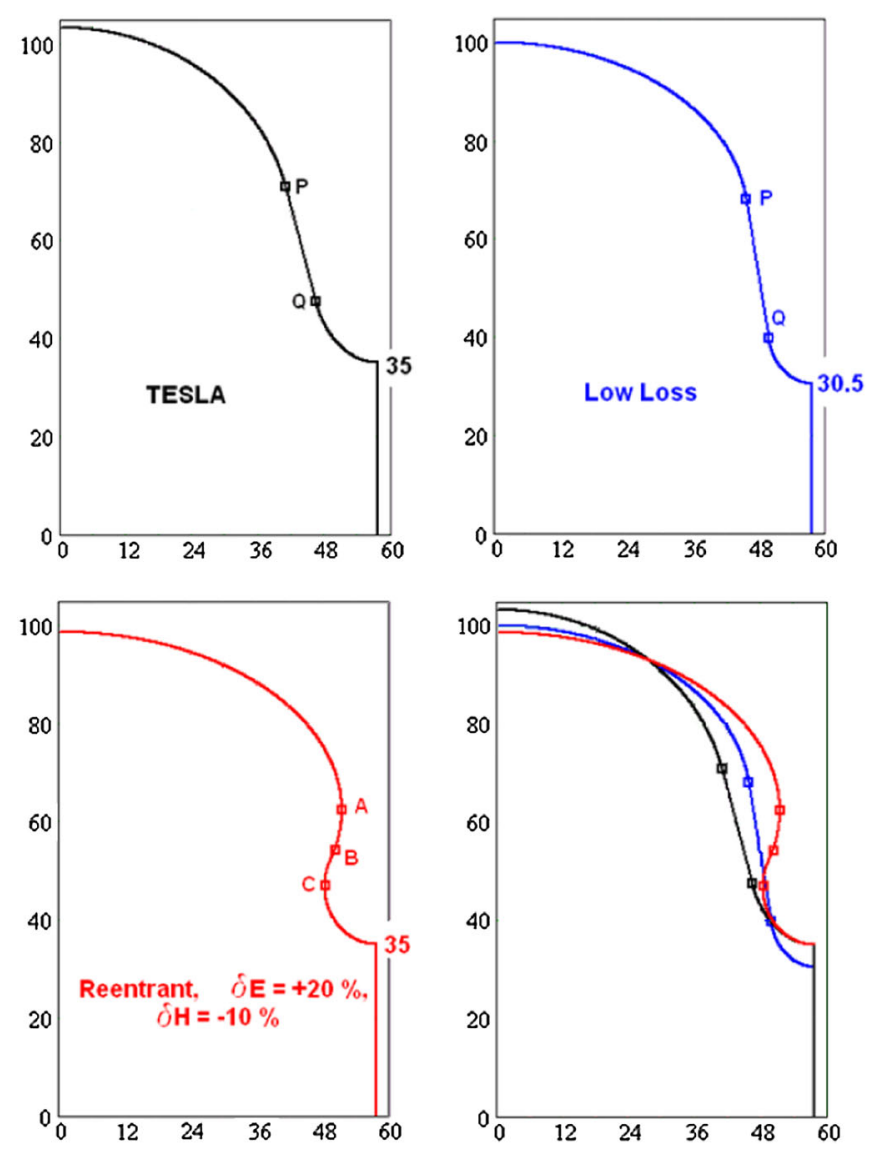

FIG. 3. Comparison of three known geometries of the elliptic half-cells.

axis of rotation and the straight segment of the wall, $l=\sqrt{\left(x_{2}-x_{1}\right)^{2}+\left(y_{2}-y_{1}\right)^{2}}$, like it was done in our earlier publications. The cavity with $\alpha<90^{\circ}$ is known as the reentrant cavity.

As an example, three widely known geometries of the inner half-cells are shown in Fig. 3: TESLA [4] from DESY, the low-loss cavity [5] from JLab, and the reentrant cavity by Cornell [6].

The surface electric and magnetic fields in these cavities are shown in Figs. 4 and 5. One can see that the electric

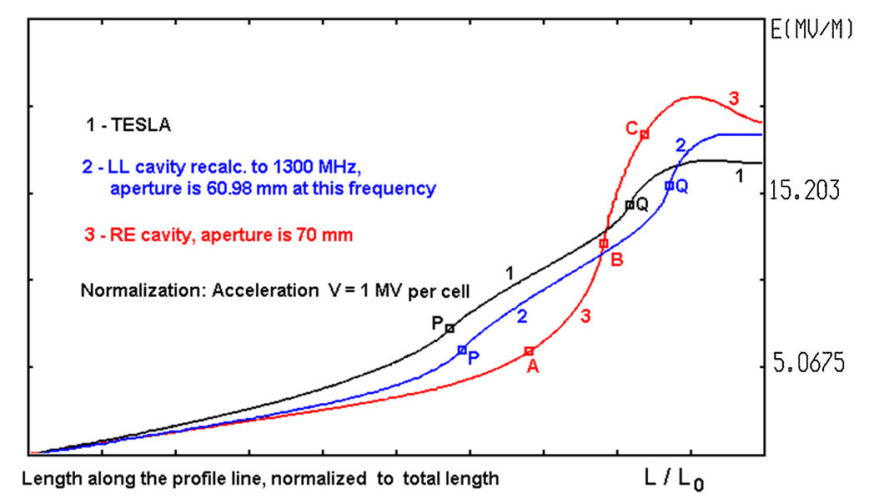

FIG. 4. Electric field on the surface of the cavities from Fig. 3.

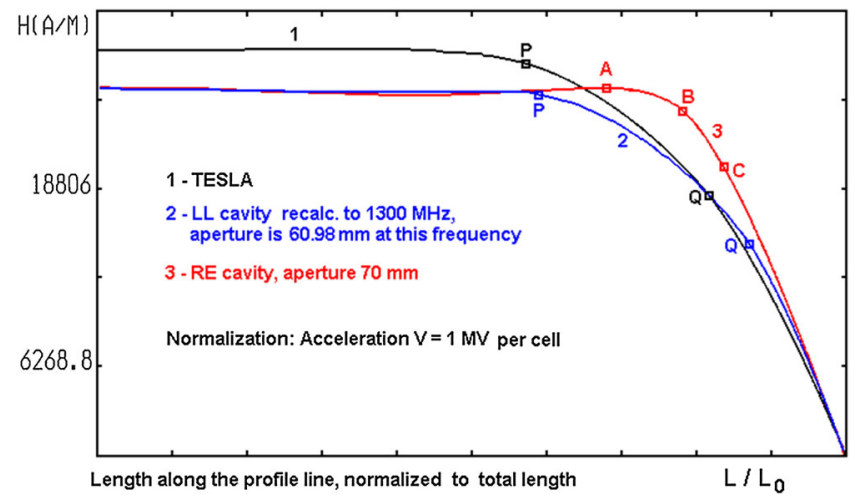

FIG. 5. Magnetic field on the surface of the cavities from Fig. 3.

field has irregularities at the points $P$ and $Q$ on the surface of non-reentrant cavities - where the arc conjugates with the segment of the straight line. We believe that the best shape should have more regular dependencies for these curves, but for this purpose we have, possibly, to abandon the paradigm of an elliptic shape and to pass to a smoother contour, e.g., using some kind of splines [7].

In the case of the reentrant cavity, the maximum is not flat as it is on non-reentrant geometry. One can suppose that flattening of this maximum will decrease the peak electric field but the $E_{\text {acc }}$ should not change significantly.

Comparing the magnetic fields of the TESLA cell and the reentrant cell having the same aperture we can see that decrease of the maximal field is obtained through the lengthening of the maximal field region. Again, better flattening of the maximal values of this field could decrease the peak field though not much. The area under these curves is approximately the same in both cases. In the case of the low loss cavity the decrease of the field is obtained due to lesser aperture. As it will be shown below, $H_{\mathrm{pk}} / E_{\mathrm{acc}}$ is a monotone function of the aperture radius and it is smaller here due to a smaller $R_{a}$.

Because of high cost of superconducting cavities even a several percent further decrease of $H_{\mathrm{pk}} / E_{\mathrm{acc}}$ is worth the investigations, and we see that this decrease is possible.

\section{SOME DEFINITIONS}

The accelerating field $E_{\text {acc }}$ is defined as the energy increment $\Delta U$, in volts, after the passage of a particle through the inner cell of the cavity to the length of this cell. For $\beta=1$ this length ( $2 L$, Figs. 2 and 3 ) is equal to $\lambda / 2$, $L=\lambda / 4$. In the case of $\beta<1$ we have to define the accelerating field as $E_{\mathrm{acc}}=\Delta U /(\beta \lambda / 2)$ since the length of the half-cell is $L=\beta \lambda / 4$. In the case when the particle velocity does not correspond to the length of the cell, the value of $\Delta U$ will differ from the value calculated for the geometrical $\beta_{\text {geo }}$. For fear of causing confusion, we will use $E_{\text {acc }}$ corresponding to the geometrical beta. Usage of the particle velocity $\beta_{\text {eff }}$ higher than $\beta_{\text {geo }}$ is justified by the fact 
that the transit time factor becomes higher than for $\beta_{\text {eff }}=$ $\beta_{\text {geo }}$ if this exceedance is about $2 \%-5 \%$.

The end cells do not have a physically defined exact length because the field propagates in the beam pipe exponentially decreasing. It appears logical for calculation of the accelerating field in the end cells to use the same definition: $E_{\text {acc }}=\Delta U /(\beta \lambda / 2)$, so that the length of this cell is not required.

It is convenient to compare different cavities with a well known one. So, for the definition of the overvoltage $E_{\mathrm{pk}} / E_{\mathrm{acc}}$ where $E_{\mathrm{pk}}$ is maximal electric field on the cavity surface, we will use the value of $e=E_{\mathrm{pk}} / E_{\mathrm{acc}} / 2$ so that for the well-known TESLA cavity $e=1$.

Analogously, the values of $H_{\mathrm{pk}} / E_{\mathrm{acc}}$ are normalized to corresponding values of TESLA $[42 \mathrm{Oe} /(\mathrm{MV} / \mathrm{m})]$ so that $h=H_{\mathrm{pk}} / E_{\mathrm{acc}} / 42$ is equal to 1 for TESLA cells. \{According to our calculations, the normalized magnetic field appears about $1.5 \%$ less than this value $[41.4 \mathrm{Oe} /(\mathrm{MV} / \mathrm{m})]$. In publications [8] of 1992 (Haebel) $H_{\mathrm{pk}} / E_{\mathrm{acc}}=41.7$, later [9] (Edwards, 1995) this value is shown as 42 , the last publication [4] (Aune, 2000) gives $42.6 \mathrm{Oe} /(\mathrm{MV} / \mathrm{m})$. Normalization for 42 is chosen because: (1) it is convenient to use round numbers, (2) some deviations in different references are about this value.\}

The dimensions of cavities (always in millimeters) unless specially noted otherwise-are given for the frequency of $1300 \mathrm{MHz}$, this is a "most popular" frequency of superconducting cavities. However, all the optimizations will be valid for any frequency because the discussed figures of merit, $E_{\mathrm{pk}} / E_{\mathrm{acc}}, H_{\mathrm{pk}} / E_{\mathrm{acc}}$, as well as $R_{\mathrm{sh}} / Q$ - the effective cavity impedance, and $G R_{\mathrm{sh}} / Q$ the geometrical factor of losses, do not depend on frequency; dimensions should be scaled only, if a different frequency is used. Here $R_{\mathrm{sh}}$ is the shunt impedance of a cavity, $Q$ is the quality factor, and $G$ is the geometrical impedance.

\section{METHOD OF OPTIMIZATION}

First of all it should be noted that for calculation of the secondary values for any geometrical parameters we used the SuPERLANS code by D. Myakishev and V. Yakovlev [10] and the TunEDCELl envelope code written by Dmitri Myakishev [11]. These codes have the necessary accuracy needed for our optimization [12].

From the previous optimization [13] for the minimum $h$ we know that this minimum is a monotone function of the overvoltage $E_{\mathrm{pk}} / E_{\mathrm{acc}}$, it is decreasing when this value increases, and a monotone function of the wall slope angle $\alpha$, it is decreasing when the angle becomes smaller. So, the minimal value of $h$ will be at the given permitted values of $E_{\mathrm{pk}} / E_{\mathrm{acc}}$ and $\alpha$.

The goal of the cavity optimization is to find this minimum of $h$ under given constraints: the value of $e$ is less than or equal to a given one, and the value of $\alpha$ is bigger

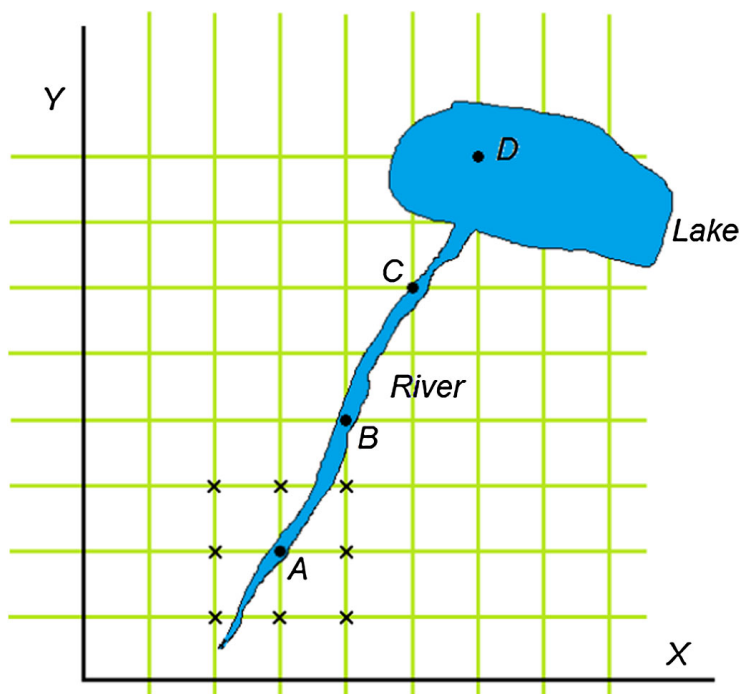

FIG. 6. Brute force optimization does not lead to the minimum.

than or equal to a given one. We are going to minimize the function $h(A, B, a, b)$ under these conditions. The "brute force" or the "grid search" approach is to calculate $h$ on a 4-dimensional grid around some point, to find the minimum of $h$ between these points, to make this point of minimum the central point and repeat the procedure. When the central point is the best point for a given steps in all 4 directions, the value of this step can be decreased, so the minimum can be found with a needed accuracy. However, the example presented in Fig. 6 shows that this method is inefficient when the optimized function belongs to a class of so called ravine functions.

In order to prove this, we tried to solve the optimization problem with the Levenberg-Marquardt algorithm [14] which is gradient based. And in fact, as predicted by literature, due to the ravine shape of the problem, it did not show good convergence compared to a Monte-Carlo based algorithm. Eventually we used a Monte-Carlo scheme that uses information from precomputed values, which is often referred to as Markov-Chain MonteCarlo in the literature [15]. Therefore we pick a starting point from which, by Monte-Carlo, a better point is searched.

At the points marked with crosses around the point $A$ (Fig. 6), the level of the surface is higher than at the point $A$. This level is decreasing to points $B, C$, and $D$. In this example steps along $Y$ should be two times bigger than in the direction $X$ to find the correct minimum.

As it will be shown below (Figs. 8-11), we have exactly the case of a ravine function: a rapid descent to the boundary and gently inclined way along the boundary.

The Monte Carlo method to find the minimum was faster than the grid method but it also "stuck" very often not reaching the final minimal point. To check the correctness of the found minima $h(A, B, a, b)$ for each value of the slope angle $\alpha$, as presented in Figs. 17 and 19 to 24, the 

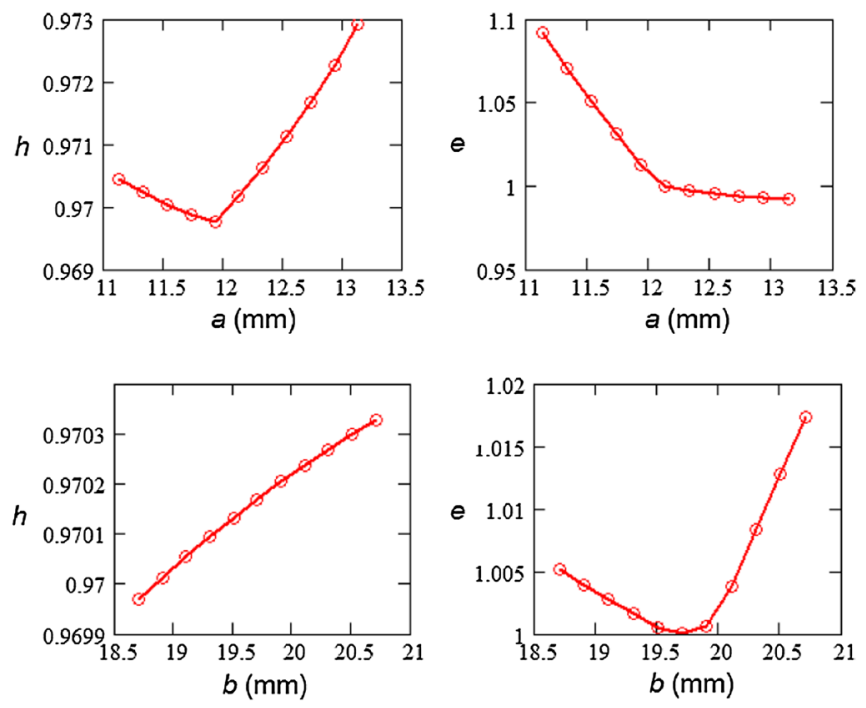

FIG. 7. Dependencies of $h$ and $e$ on $a$ and $b$ near the point of $\min h$.

dependences of $A, B, a$, and $b$ on $\alpha$ were plotted, an example is in Fig. 18. Very often these dependences appeared to be not smooth, in these cases a more thorough search for the minimum was needed.

In the search for the minimum $h$ we can easily come to the boundary where the limiting value of $e$ and $\alpha$ are reached. To move along the "bottom" of the ravine we can solve the system of equations:

$$
\begin{aligned}
& \partial h / \partial A \cdot \Delta A+\partial h / \partial B \cdot \Delta B+\partial h / \partial a \cdot \Delta a \\
& \quad+\partial h / \partial b \cdot \Delta b=\Delta h, \\
& \partial e / \partial A \cdot \Delta A+\partial e / \partial B \cdot \Delta B+\partial e / \partial a \cdot \Delta a \\
& \quad+\partial e / \partial b \cdot \Delta b=\Delta e=0, \\
& \partial \alpha / \partial A \cdot \Delta A+\partial \alpha / \partial B \cdot \Delta B+\partial \alpha / \partial a \cdot \Delta a \\
& \quad+\partial \alpha / \partial b \cdot \Delta b=\Delta \alpha=0 .
\end{aligned}
$$

Here, $\Delta h$ is a negative value to be added to $h$ to decrease it.

Now, we have three equations and four unknowns: $\Delta A$, $\Delta B, \Delta a, \Delta b$. The fourth equation can be added if we want to go to the minimum by the shortest way, so that the length of the vector of increments is minimal:

$$
\Delta A^{2}+\Delta B^{2}+\Delta a^{2}+\Delta b^{2}=s^{2} .
$$

For any $\Delta h$ we can choose $s$, preferably small and solve this system of equations. So, we can consider this approach as an implementation of the gradient method in the case of the ravine function and the function under some constraints.

We can solve the system of equations (3) and (4) for any negative value of $\Delta h$ and find a lower point than the point we started of. It looks like we can decrease $h$ indefinitely long time. Of course, this is possible only as long as the absolute value of $\Delta h$ is small enough so that the partial derivatives do not change significantly.

Nevertheless, it is not clear from this system that the minimum is reached. It becomes clear if we consider the dependencies of $h$ or $e$ on $A, B, a$, and $b$. In Fig. 7 the dependencies of $h$ and $e$ on $a$ and $b$ are shown for the case of $\beta=1, R_{a}=35 \mathrm{~mm}, e=2, \alpha=100^{\circ}$ (see this point in Table II and Fig. 17). The minimum $h$ appears near the point of break of this dependence. When the derivative is considered for the left side of the point of break $(\Delta a<0)$, the result of solution of the system (3)-(4) gives $\Delta a>0$, and vice versa, considering $\Delta a>0$, the solution gives $\Delta a<0$. This contradiction shows that further decrease of $h$ is impossible, so the minimum is reached.

The same consideration can be done for all other partial derivatives in (3). However, in practice, only derivatives of $h$ and $e$ with respect to $a$ and $b$ can change their behavior near the point of minimum $h$.

The cause of this break is the following: there are two local maxima on the curves of $H$ vs $L / L_{0}$, Fig. 5; one maximum is at $L=0$, another one is in the right part of each curve, like the maximum near the $A$ point in the RE cavity. There can be also two local maxima on the flat part of the curve $E$ vs $L / L_{0}$, Fig. 4. The second local maximum $E$ at $L / L_{0}=1$ appears by thorough optimization and practically cannot be seen on the flat part of the curve 2 , Fig. 4. These maxima are not very well pronounced on these graphs because the curves become very flat between them in the process of optimization. When changing, for example, the half-axis $a$, trying to minimize $H_{\mathrm{pk}}$ (and so the $h$ ), the bigger maximum of $H$ decreases but the smaller one grows and can overtake the other one. At the point of break (Fig. 7), these maxima become equal, and if we continue to change $a$, the value of $H_{\mathrm{pk}}$ is defined now by the other hump and its dependence on $a$ changes. So, the minimum $h$ is reached when the humps on the curve $H$ vs $L / L_{0}$ or on the curve $E$ vs $L / L_{0}$ become equal by height. In the last case the decrease of $h=H_{\mathrm{pk}} / E_{\mathrm{acc}}$ due to equalization of local maxima $E$ becomes not because of minimization of $H_{\mathrm{pk}}$ but because of increase of $E_{\mathrm{acc}}$ when the force lines are reaching their maximum uniformly around the iris tip.

\section{MORE CONSTRAINTS TO THE SHAPE OF THE ELLIPTIC CAVITY}

The proposed optimization can lead to shapes that are not realizable as an inner cell of a multicell cavity. So, for the reentrant cavity, and in most of cases for the non-reentrant cavity (except of the case of very big half-axes $B$ ), the value of $A$ cannot be bigger than the cavity length. Moreover, taking into account the thickness of the wall, usually $3 \mathrm{~mm}$, the restriction $A+3<L$ should be applied. If we consider 
the necessary gap between cells needed for welding them together, we should make this restriction even more severe. However, this restriction is valid for the frequency of $1300 \mathrm{MHz}$. If the same thickness $3 \mathrm{~mm}$ is used for a cavity, say, $650 \mathrm{MHz}$, the restriction due to the touch of the walls becomes $A+1.5<L$. Here $A$ and $L$ are parameters of cavity at $1300 \mathrm{MHz}$ (not at $650 \mathrm{MHz}$ ). So, the scaling factor should be taken into account. The absolute restriction is $A<L$.

Nowadays, the cavities are mainly stamped from niobium sheets. To guarantee accuracy in the process of stamping the half-cells, the curvature radius of the iris cannot be too small even if it does not increase the $E_{\mathrm{pk}}$. The reasonable minimal value of this radius is twice the thickness of the niobium sheet used in fabrication (usually $3 \mathrm{~mm}$ ): $r_{c}>6 \mathrm{~mm}$. In case of the frequency different from $1300 \mathrm{MHz}$, the scaling should be also taken into account.

Even if the curvature is bigger than $6 \mathrm{~mm}(f=$ $1300 \mathrm{MHz}$ ), the value of $a$ cannot be smaller than $3 \mathrm{~mm}$ because the outer surface of the iris should be reachable. This gap should be possibly several millimeters wide to make possible welding of the cells.

For example the TRASCO-ASH cavity for $\beta=0.47$ and frequency $f=704.4 \mathrm{MHz}$ [16] has the radius of curvature at the iris tip $r_{c}=6.1 \mathrm{~mm}$ that corresponds to $r_{c}=$ $3.31 \mathrm{~mm}$ at $f=1300 \mathrm{MHz}$. The niobium sheet thickness for the cavities described in this paper was ranging from 3.3 to $4.3 \mathrm{~mm}$.

\section{AN EXAMPLE OF OPTIMIZATION FOR THE TESLA CAVITY}

The TESLA cavity $[4,9]$ is thoroughly studied because it was proposed to be used in the International Linear Collider. In spite of "round" values of the elliptical halfaxes $(A=B=42, a=12, b=19 \mathrm{~mm})$ it is very close to the optimized one in the way of optimization proposed here. In Fig. 8 are presented the values of normalized parameters $h$ and $e$, and the wall slope angle $\alpha$ for fixed values of $a=12 \mathrm{~mm}$ and $b=19 \mathrm{~mm}$ and for different values of $A$ and $B$ around the nominal point. The pink area for $e$ and the blue area for $\alpha$ are "forbidden" areas, i.e., areas beyond the limiting values of $e=0.997$ and $\alpha=103.17^{\circ}$. (We call these areas "forbidden" in the sense described in Sec. IV: to find the minimal $h$ for $e$ less than or equal to a given one and $\alpha$ bigger than or equal to a given one, so the values $e$ and $\alpha$ violating these inequalities are "forbidden." Also see the second paragraph in this section). On the lower right picture all three parameters are presented, "twice forbidden" area is colored green. One can see that there is a possible improvement of the value of $h$ from $h=0.99$, being in the white area.

After further optimization we will come to the Fig. 9. We have chosen the angle $\alpha=100^{\circ}$ that is a little bit less than in the original cavity $\left(103.17^{\circ}\right)$ and have chosen $e=1$ (we also prefer "round" values.) Both of these changes
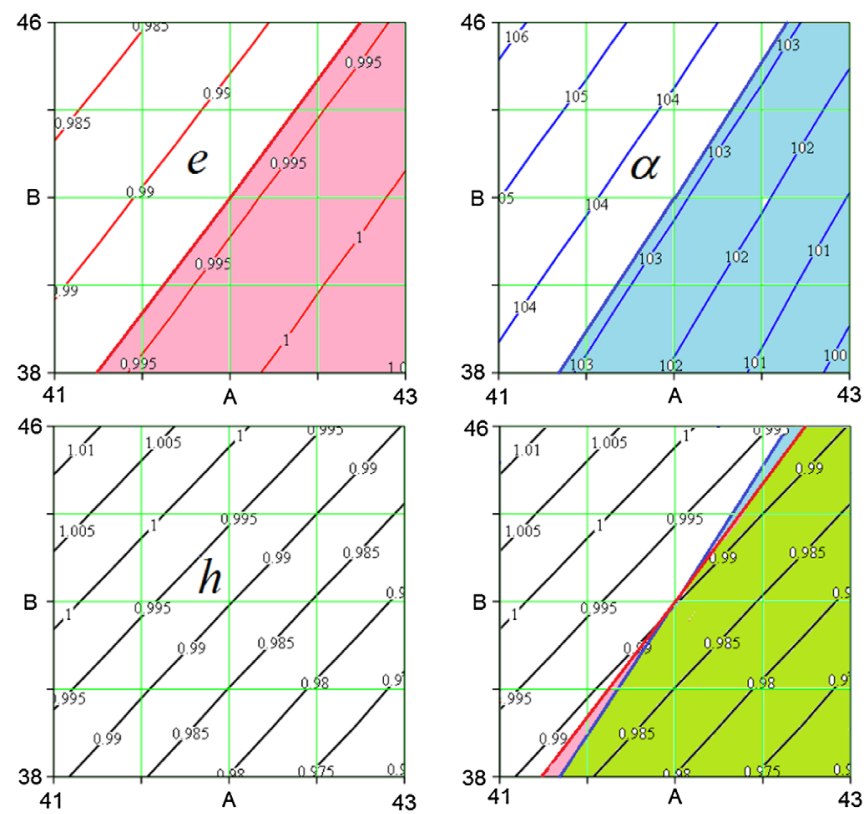

FIG. 8. Map for the original TESLA cavity. $a=12 \mathrm{~mm}$, $b=19 \mathrm{~mm}, \beta=1$.

improve the value of $h$ from 0.99 to 0.97 , but this is not the main result.

We believe that the main result consists in the fact that moving along the colored line and staying in the white area, we can change both $A$ and $B$ in a wide range changing the value of $h$ not more than $0.5 \%$. As will be shown later, this gives a possibility to create a geometry of a cavity, practically optimal, but free of multipactor or tune off the dangerous higher order modes.
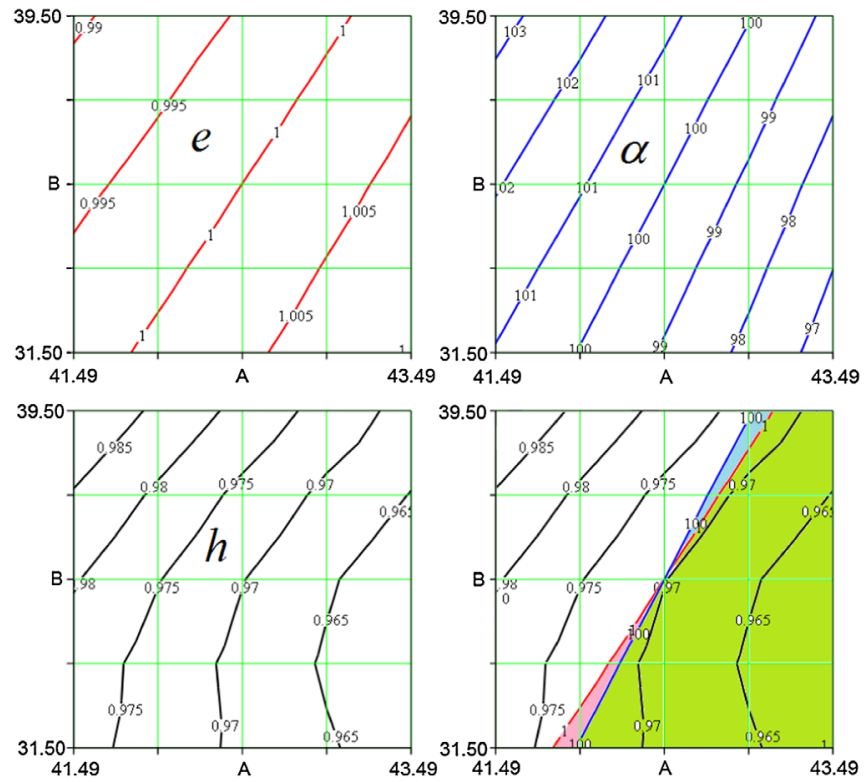

FIG. 9. Map for the optimized TESLA cavity. $a=12.13 \mathrm{~mm}$, $b=19.70 \mathrm{~mm}, \beta=1$. 
One can understand that this fact of a very flat minimum where some values can be changed in a broad range is a reverse side of the difficulty to find an exact minimum as described in the section "Method of optimization."

\section{AN EXAMPLE OF OPTIMIZATION FOR THE SNS ELLIPTIC CAVITY WITH $\beta_{\mathrm{geo}}=0.81$}

As an example of an elliptic cavity with $\beta=0.81$ let us analyze the shape and fields of the Spallation Neutron Source (SNS) cavity [16]. According to the data from this paper the inner half-cell of the cavity for frequency $f=805 \mathrm{MHz}$ has the wall distance $d=15 \mathrm{~mm}$, wall angle $\gamma=7^{\circ}$, length $L=75.5 \mathrm{~mm}$, aperture radius $R_{a}=48.8 \mathrm{~mm}, \beta_{\text {geo }}=0.81$ and $\beta_{\text {eff }}=0.83$. The values of normalized peak fields are reported as $E_{\mathrm{pk}} / E_{\mathrm{acc}}=2.19$ and $B_{\mathrm{pk}} / E_{\mathrm{acc}}=4.72 \mathrm{mT} /(\mathrm{MV} / \mathrm{m})$.

From these data, using formulas (1) and (2) we calculated the half-axes of ellipses and the normalized peak fields: $A=B=52.376 \mathrm{~mm}, \quad a=18.677, \quad b=33.619 \mathrm{~mm}$, $E_{\mathrm{pk}} / E_{\mathrm{acc}}=2.145$, and $B_{\mathrm{pk}} / E_{\mathrm{acc}}=4.572$. The equatorial radius was calculated by tuning the cavity to the given frequency.

Our results are $2.3 \%$ lower for the $E_{\mathrm{pk}} / E_{\mathrm{acc}}$ and $3.3 \%$ lower for the $B_{\mathrm{pk}} / E_{\mathrm{acc}}$ value. These values were calculated for $\beta_{\text {geo }}=0.81$. If $\beta_{\text {eff }}=0.83$ is used, the deviation of $E_{\mathrm{pk}} / E_{\mathrm{acc}}$ and $B_{\mathrm{pk}} / E_{\mathrm{acc}}$ from the values presented in the paper is even bigger: $E_{\mathrm{pk}} / E_{\mathrm{acc}}=2.1228$ and $B_{\mathrm{pk}} / E_{\mathrm{acc}}=$ 4.519 , or $e=1.0614$ and $h=1.076$.

This discrepancy can be explained by rounded values of geometrical parameters given in the paper (we do not have direct values of half-axes $A, B, a$, and $b$ ), we also believe that the software SUPERLANS used in our calculations has better accuracy [12] than SUPERFISH used in the cited work.

We will perform the optimization for the effective value of beta, because the used beam has this value of speed. For further analysis we will scale the dimensions of this cavity from $805 \mathrm{MHz}$ to $1300 \mathrm{MHz}$. Let us analyze first the original geometry after scaling. In Fig. 10 are presented the values of normalized parameters $h$ and $e$, and the wall slope angle $\alpha$ for fixed values of $a$ and $b$ and different values of $A$ and $B$. The pink area for $e$ and the blue area for $\alpha$ are "forbidden" areas, i.e., areas beyond the limiting values of $e=1.0614$ and $\alpha=97^{\circ}$. On the lower right picture all three parameters are presented, "twice forbidden" area is colored green. One can see that there is a possible improvement of the value of $h$ from $h=1.076$, being in the white area.

Figure 11 shows the same parameters after optimization using the same limiting values of $e$ and $\alpha$. The value of $h$ is improved to 1.069 , though not so much: $0.6 \%$. But the more interesting feature of this result, as well as in the previous section, is the fact that moving from the point near the lower left corner to the point near the upper right corner
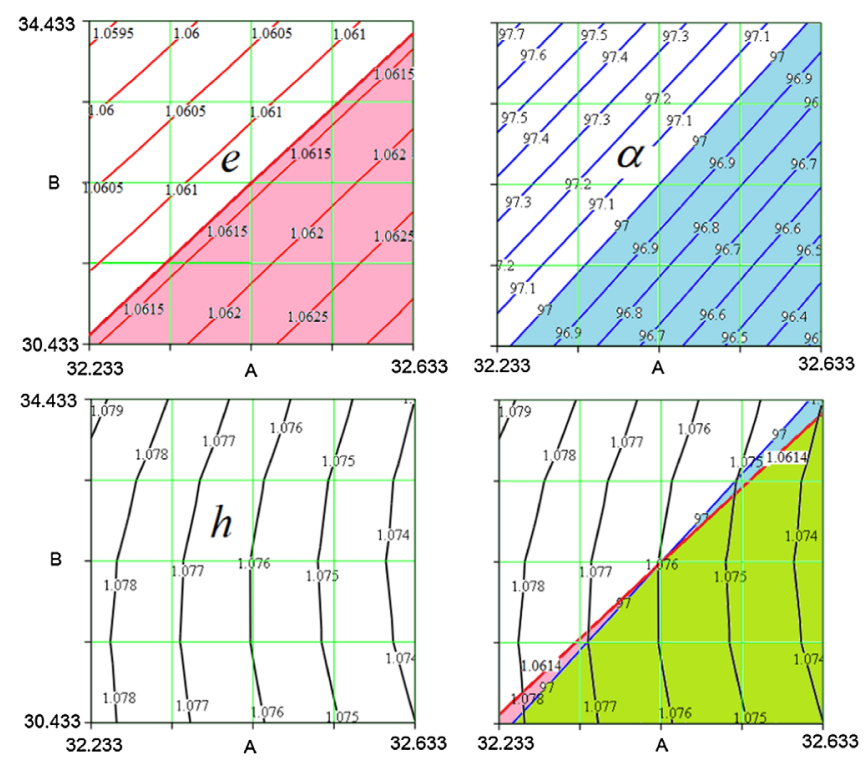

FIG. 10. Map for the original SNS cavity. $a=11.565 \mathrm{~mm}$, $b=20.818 \mathrm{~mm}, \beta=0.81$.
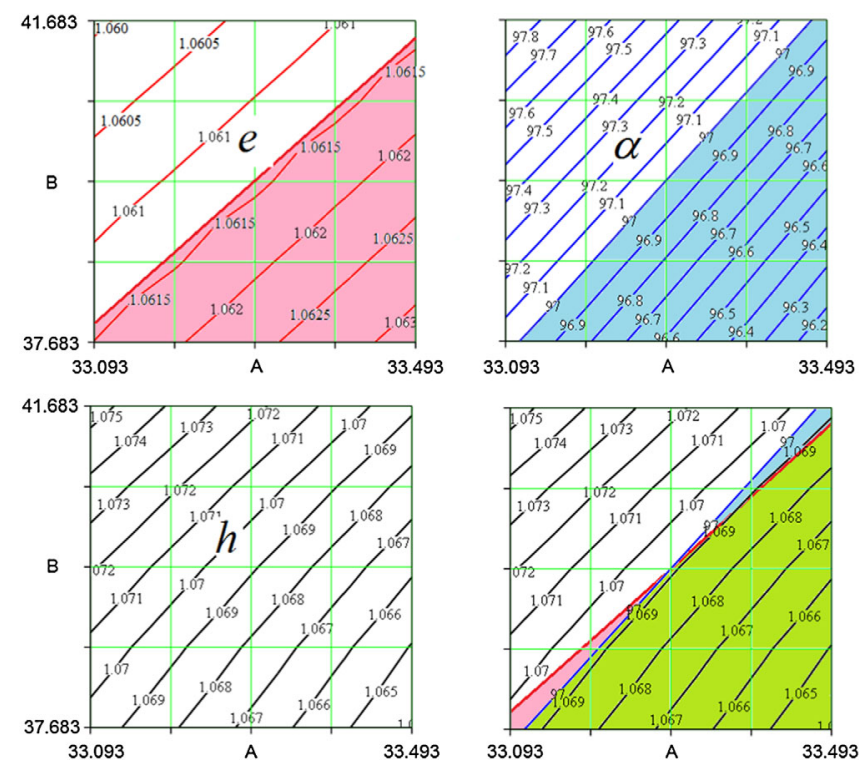

FIG. 11. Map for the optimized SNS cavity. $a=11.387 \mathrm{~mm}$, $b=21.009 \mathrm{~mm}$.

and remaining in the noncolored area we will have the value of $h$ practically the same as at the point of optimum (it is in the center) but changing significantly the values of $A$ and $B$. These changes give us freedom to avoid multipactor or/and to tune the most dangerous higher order modes to a nondangerous position being near the lowest possible value of $h$, i.e., lowest magnetic field and lowest losses.

\section{MULTIPACTOR CONSIDERATION}

Multipactor is a parasitic of phenomenon limiting the accelerating rate of a cavity. There are several varieties of 


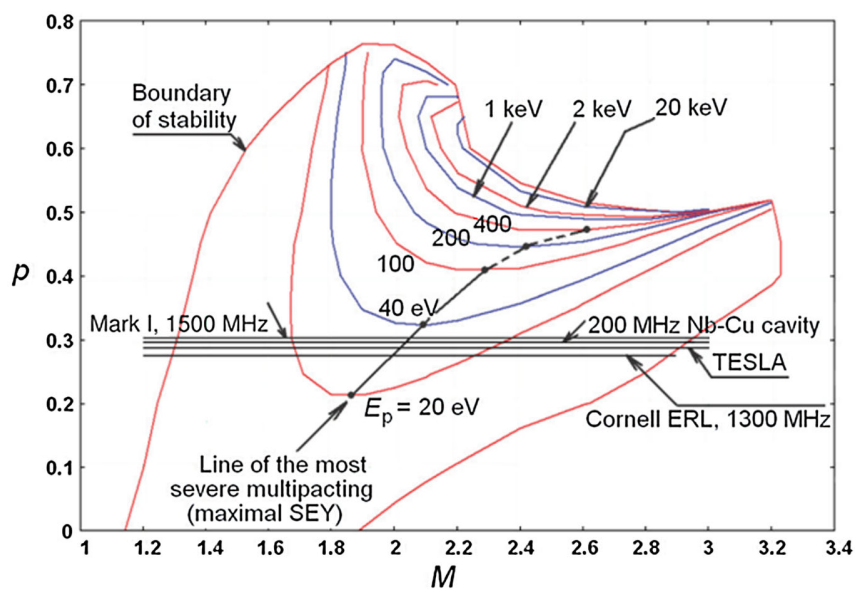

FIG. 12. Equatorial cross-field multipacting zone of the first order.

multipactor depending on the geometry of an rf device. For a superconducting elliptical cavity the 2-point multipactor on the cavity equator occurs most often. We have a method of predicting the appearance of this type of multipactor without time consuming simulations but immediately from the field calculations done with a 2D program [17].

Zone of existence of multipactor on the cavity equator, Fig. 12, is defined by two coefficients: the geometrical coefficient $p=\beta /(\alpha+\beta)$ and the field coefficient $M=e B_{0} / m \omega$, where $e / m$ is the specific charge of the electron, $\omega=2 \pi f$ is the angular frequency of oscillations and $\alpha$ and $\beta$ are factors of proportionality for the longitudinal and radial components of the electric field near the equator, $E_{z}=\alpha d$ and $E_{r}=\beta d$, respectively. The points of definition of these factors are at a distance $d=1 \mathrm{~mm}$, Fig. 13, this is a characteristic size for the orbit of an electron in this kind of multipactor at any frequency as follows from [17]. For a frequency lower than $f=$ $1300 \mathrm{MHz}$ this value of $d$ can be proportionally increased to have a better accuracy of the calculated fields. Actually, $p$ weakly depends on $d$ because the area of linearity for $E_{z}$ and $E_{r}$ is bigger than $1 \mathrm{~mm}$ at $f=1300 \mathrm{MHz}$.

The value of $B_{0}$ in the expression for the field coefficient $M$ is the amplitude of the magnetic field at the equator where the multipactor takes place. $B_{0}$ is proportional to the accelerating field $E_{\text {acc }}$ in the cavity and usually is maximal or close to the maximal magnetic field in the cavity, $B_{\mathrm{pk}}$.

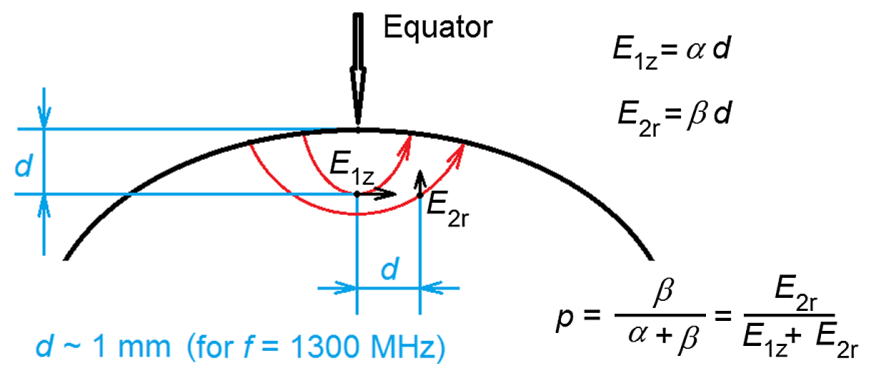

FIG. 13. Definition of the geometrical parameter $p$.
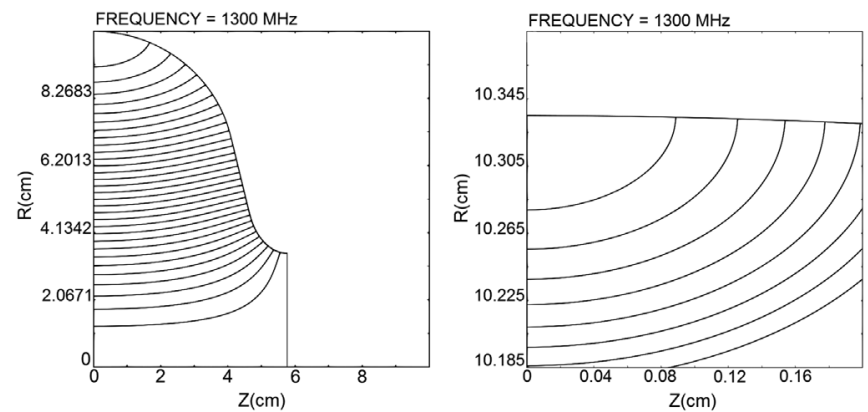

FIG. 14. Electric force lines for the entire half-cell of the TESLA cavity and near the equator. Force lines near the equator are elliptic arcs with ratio of half-axes $b_{\mathrm{fl}} / a_{\mathrm{fl}}=0.633<0.655$, see text.

The value of $B_{\mathrm{pk}} / E_{\mathrm{acc}}$ is always known when the cavity is being designed. So, knowing $B_{0}$ when the multipactor starts, we can easily find $E_{\text {acc }}$ for this condition.

Each cavity has its own geometrical coefficient $p$. Moving along the line $p=$ const, as shown in Fig. 12, we can increase the magnetic field at the point of the equator, i.e., the field coefficient $M$. When the energy of the primary electrons $E_{p}$ (see the graph) becomes high enough for the secondary emission yield $>1$, the multipactor starts. This happens usually near the point $M=2$ as can be seen from the figure. The results with different cavities analyzed in the paper [17] and presented in Fig. 12 show that the critical value for multipactor is about $p=0.3$, so that we have a very weak and usually easily processed multipactor in the TESLA cavity ( $p=0.286$ ), a well pronounced multipactor in the Mark I cavity fabricated specially for studying this phenomenon $(p=0.303)$, and practically no multipactor at the equator of the Cornell ERL cavity $(p=0.276)$. More details and references can be found in the cited paper.

The force lines of the electric field near the equator have a shape of elliptic arcs (Fig. 14) as follows from

$$
\frac{E_{r}}{E_{z}}=\frac{d r}{d z}=\frac{-\beta z}{\alpha r}
$$

here $r$ is measured from the equator to the axis.
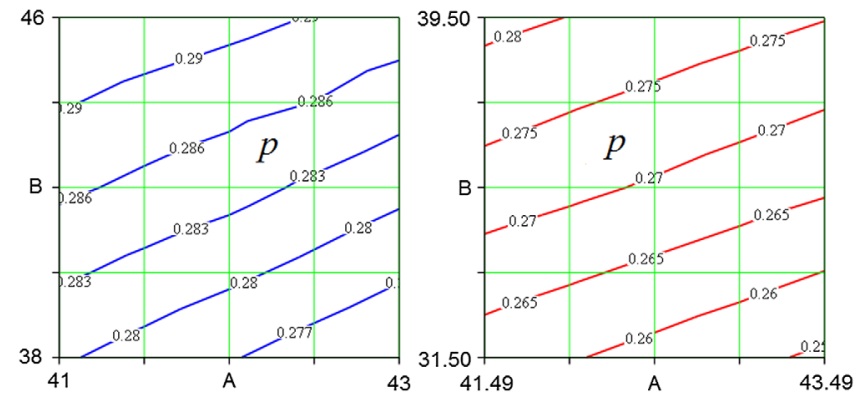

FIG. 15. Parameter $p$ for the TESLA cavity, original (left) and optimized, in the same coordinates as in Figs. 8 and 9. 


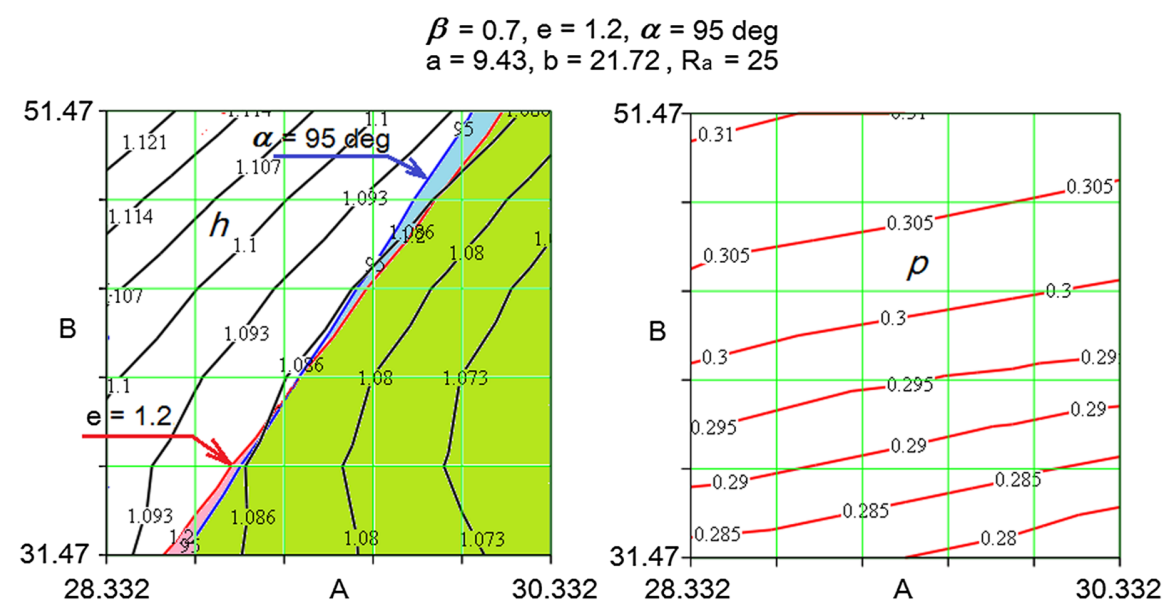

FIG. 16. Map for one of the points from Fig. 21 for $\beta=0.7$. Wide choice of $A$ and $B$ keeping the optimal $h$. A possibility to get rid of multipactor choosing $p<0.3$.

The solution of this differential equation is

$$
\frac{z^{2}}{\alpha}+\frac{r^{2}}{\beta}=\text { const. }
$$

So, the ratio of the half-axes of the elliptic force lines near the equator is $b_{\mathrm{fl}} / a_{\mathrm{fl}}=\sqrt{\beta / \alpha}=\sqrt{p /(1-p)}$ and the condition $p>0.3$ corresponds to $b_{\mathrm{fl}} / a_{\mathrm{fl}}>\sqrt{3 / 7} \approx 0.655$.

As it was stated above in the section "An example of optimization of the TESLA cavity," we can change the dimensions $A$ and $B$ so that the value of normalized magnetic field $h$ will only slightly change in a wide range of $A$ and $B$. For the example presented in Figs. 8 and 9, let us present values of $p$ in the same coordinates, Fig. 15. We will not overlap this additional graph with the previous ones but it is easily seen that (i) the optimized cavity has significantly decreased $p$, so that there will be no multipacting, and (ii) this parameter can be further decreased increasing the value of $h$ not more than $0.5 \%$.

\section{HIGHER ORDER MODES OF THE MODIFIED SHAPES}

To avoid the higher order modes (HOM) cavity frequencies falling on dangerous frequency spectrum lines of beam is an important design task for the ERL cavity designer. The presented approach to change the cavity shape keeping the optimal value of $H_{\mathrm{pk}} / E_{\mathrm{acc}}$ for given $E_{\mathrm{pk}} / E_{\mathrm{acc}}, R_{a}$, and $\alpha$ will also change the spectrum of the cavity HOMs. We calculated the lowest monopole and dipole modes of the TESLA cavity when the bigger half-axes of it are changed from 42 to $41.1 \mathrm{~mm}$ for $A$ and from 42 to $38 \mathrm{~mm}$ for the $B$ half-axis. According to Fig. 8 the value of $h$ will not change compared to the original shape. The data are presented in Table I. These data are calculated for an infinitely long multicell cavity consisting of identical cells.

One can see that the change in frequencies is present though not so big as wished it to be. However, the range of changes of these half-axes can be increased, so will be increased the shift of the HOMs.

\section{TABLES AND GRAPHS FOR DIFFERENT VALUES OF $\beta$}

\section{A. Elliptic cavities with $\beta=1$}

Results of optimization for $\beta=1$ are presented in Table II and in Figs. 17 and 18. Curves in Fig. 18 are plotted in accordance to data in Table II.

\section{B. Elliptic cavities with $\beta=0.9$}

Results of optimization for $\beta=0.9$ are presented in Fig. 19 and Table III.

TABLE I. Frequencies of higher order modes for geometries specified in Fig. 8: original TESLA cavity and its modification without increasing magnetic field. Smaller elliptic half-axes are kept same: $a=12 \mathrm{~mm}, b=19 \mathrm{~mm}$. Bigger half-axes $A$ and $B$ are seen in the Table.

\begin{tabular}{lcc}
\hline \hline $\begin{array}{l}\text { Geometry, dimensions } \\
\text { in mm }\end{array}$ & $\begin{array}{c}A=42 \mathrm{~mm}, \\
B=42 \mathrm{~mm}\end{array}$ & $\begin{array}{c}A=41.1 \mathrm{~mm}, \\
B=38 \mathrm{~mm}\end{array}$ \\
\hline Lowest monopoles, $\mathrm{MHz}$ & 1275.7 & 1275.7 \\
& 1300 & 1300 \\
& 2672.0 & 2675.5 \\
& 2774.4 & 2778.9 \\
& 3757.2 & 3758.0 \\
Lowest dipoles, $\mathrm{MHz}$ & 3857.8 & 3865.5 \\
& 1792.4 & 1792.6 \\
& 1888.3 & 1888.5 \\
& 2471.9 & 2472.5 \\
& 3124.6 & 3125.3 \\
& 3343.9 & 3353.5 \\
& 3361.6 & 3371.3 \\
\hline \hline
\end{tabular}


TABLE II. Values of $H_{\mathrm{pk}} / E_{\mathrm{acc}}, G R / Q$ and dimensions of optimized inner cell (two elliptic arcs and a straight segment) vs the slope angle $\alpha$. Optimization for $\min H_{\mathrm{pk}} / E_{\mathrm{acc}}($ or $\min h)$.

\begin{tabular}{|c|c|c|c|c|c|c|c|c|}
\hline \multicolumn{9}{|c|}{ Optimization for $\min h, \beta=1$} \\
\hline \multicolumn{9}{|c|}{$R_{a}=35 \mathrm{~mm}, E_{\mathrm{pk}} / E_{\mathrm{acc}}=2(e=1)$} \\
\hline \multicolumn{2}{|c|}{$\begin{array}{l}\text { Normalized peak magnetic field } \\
\text { (also norm. to } 42 \text { ) }\end{array}$} & \multicolumn{6}{|c|}{ Geometrical dimensions in $\mathrm{mm}$} & \multirow{2}{*}{$\begin{array}{l}\text { Loss parameter (also } \\
\text { normalized to TESLA) } \\
G R / Q, \mathrm{Ohm}^{2} ;(g r / q)\end{array}$} \\
\hline$\alpha^{\circ}$ & $H_{\mathrm{pk}} / E_{\mathrm{acc}}, \mathrm{Oe} /(\mathrm{MV} / \mathrm{m}) ;(h)$ & $A$ & $B$ & $a$ & $b$ & $l$ & $R_{\text {eq }}$ & \\
\hline 82.70 & $39.90(0.9500)$ & 45.36 & 36.19 & 12.81 & 21.35 & 0 & 100.683 & $32077(1.041)$ \\
\hline 85 & $39.91(0.9502)$ & 45.33 & 36.03 & 12.78 & 21.57 & 2.45 & 100.693 & $32068(1.041)$ \\
\hline 90 & $40.00(0.9523)$ & 44.94 & 35.65 & 12.71 & 21.48 & 8.71 & 100.833 & $32015(1.039)$ \\
\hline 95 & $40.23(0.9579)$ & 43.99 & 35.06 & 12.53 & 20.95 & 15.73 & 101.205 & $31838(1.034)$ \\
\hline 100 & $40.74(0.9701)$ & 42.49 & 35.50 & 12.13 & 19.70 & 22.76 & 102.024 & $31420(1.020)$ \\
\hline 105 & $41.78(0.9942)$ & 39.94 & 34.81 & 11.57 & 18.02 & 31.22 & 103.116 & $30599(0.993)$ \\
\hline \multicolumn{9}{|c|}{$R_{a}=35 \mathrm{~mm}, E_{\mathrm{pk}} / E_{\mathrm{acc}}=2.2(e=1.1)$} \\
\hline 74.01 & $38.60(0.9191)$ & 49.02 & 35.65 & 10.49 & 15.46 & 0 & 99.398 & $33296(1.081)$ \\
\hline 80 & $38.64(0.9201)$ & 48.81 & 35.59 & 10.50 & 15.25 & 5.43 & 99.469 & $33292(1.081)$ \\
\hline 85 & $38.75(0.9226)$ & 48.30 & 34.96 & 10.45 & 14.95 & 10.70 & 99.594 & $33232(1.079)$ \\
\hline 90 & $38.95(0.9274)$ & 47.41 & 33.59 & 10.24 & 14.73 & 16.50 & 99.810 & $33067(1.074)$ \\
\hline 95 & $39.32(0.9363)$ & 45.87 & 31.95 & 9.94 & 14.18 & 22.92 & 100.252 & $32748(1.063)$ \\
\hline 100 & $39.96(0.9515)$ & 43.63 & 31.08 & 9.56 & 13.35 & 29.22 & 101.118 & $32181(1.045)$ \\
\hline 105 & $40.95(0.9751)$ & 41.14 & 33.38 & 8.99 & 11.84 & 34.66 & 102.673 & $31247(1.015)$ \\
\hline \multicolumn{9}{|c|}{$R_{a}=35 \mathrm{~mm}, E_{\mathrm{pk}} / E_{\mathrm{acc}}=2.4(e=1.2)$} \\
\hline 67.79 & $37.79(0.8997)$ & 51.54 & 36.24 & 9.18 & 11.90 & 0 & 98.711 & $34064(1.106)$ \\
\hline 70 & $37.80(0.8997)$ & 51.46 & 36.21 & 9.22 & 11.85 & 2.68 & 98.722 & $34064(1.106)$ \\
\hline 75 & $37.83(0.9006)$ & 51.37 & 36.10 & 9.10 & 11.84 & 5.47 & 98.801 & $34043(1.105)$ \\
\hline 80 & $37.92(0.9028)$ & 51.11 & 35.11 & 8.88 & 11.96 & 9.94 & 98.838 & $33950(1.102)$ \\
\hline 85 & $38.09(0.9069)$ & 50.34 & 34.07 & 8.78 & 11.60 & 15.06 & 99.010 & $33842(1.099)$ \\
\hline 90 & $38.37(0.9135)$ & 49.10 & 32.36 & 8.55 & 11.22 & 20.71 & 99.285 & $33603(1.091)$ \\
\hline 95 & $38.84(0.9248)$ & 47.17 & 30.52 & 8.29 & 10.62 & 26.66 & 99.797 & 33199 (1.078) \\
\hline 100 & $39.54(0.9413)$ & 44.50 & 28.78 & 7.91 & 9.78 & 32.90 & 100.622 & 32568 (1.057) \\
\hline 105 & $40.59(0.9664)$ & 41.36 & 29.36 & 7.48 & 9.00 & 38.29 & 102.098 & $31579(1.025)$ \\
\hline \multicolumn{9}{|c|}{$R_{a}=30 \mathrm{~mm}, E_{\mathrm{pk}} / E_{\mathrm{acc}}=2.0(e=1.0)$} \\
\hline 77.08 & $36.62(0.8720)$ & 48.73 & 38.05 & 10.39 & 17.59 & 0 & 98.684 & 37081 (1.204) \\
\hline 80 & $36.63(0.8722)$ & 48.70 & 38.05 & 10.34 & 17.63 & 2.83 & 98.753 & 37072 (1.204) \\
\hline 85 & $36.71(0.8740)$ & 48.36 & 37.57 & 10.27 & 17.46 & 8.74 & 98.845 & 37011 (1.202) \\
\hline 90 & $36.90(0.8787)$ & 47.53 & 36.55 & 10.12 & 16.97 & 15.56 & 99.073 & 36829 (1.196) \\
\hline 95 & $37.32(0.8885)$ & 46.01 & 35.48 & 9.86 & 16.14 & 22.74 & 99.599 & 36395 (1.182) \\
\hline 100 & $38.02(0.9052)$ & 43.87 & 35.54 & 9.39 & 14.67 & 29.77 & 100.604 & 35605 (1.156) \\
\hline 105 & $39.32(0.9361)$ & 40.90 & 35.82 & 8.68 & 12.60 & 37.62 & 102.012 & 34327 (1.115) \\
\hline \multicolumn{9}{|c|}{$R_{a}=30 \mathrm{~mm}, E_{\mathrm{pk}} / E_{\mathrm{acc}}=2.2(e=1.1)$} \\
\hline 70.02 & $35.64(0.8486)$ & 51.75 & 38.48 & 8.76 & 13.10 & 0 & 97.874 & $38237(1.241)$ \\
\hline 80 & $35.75(0.8512)$ & 51.39 & 37.71 & 8.57 & 13.04 & 9.14 & 97.982 & $38129(1.238)$ \\
\hline 90 & $36.22(0.8623)$ & 49.39 & 35.00 & 8.26 & 12.07 & 21.36 & 98.427 & $37648(1.222)$ \\
\hline 100 & $37.54(0.8939)$ & 44.71 & 32.55 & 7.53 & 10.06 & 34.39 & 100.027 & $36160(1.174)$ \\
\hline 105 & $38.79(0.9236)$ & 41.83 & 35.17 & 6.90 & 8.60 & 39.81 & 101.771 & $34742(1.128)$ \\
\hline
\end{tabular}

\section{Elliptic cavities with $\boldsymbol{\beta}=\mathbf{0 . 8}$}

Results of optimization for $\beta=0.8$ are presented in Fig. 20 and in Table IV.

\section{Elliptic cavities with $\boldsymbol{\beta}=\mathbf{0 . 7}$}

Results of optimization for $\beta=0.7$ are presented in Fig. 21 and in Table V.
Figure 16 shows for one of the points from Fig. 21 that the choice of half-axes $A$ and $B$ can also be done in a very large range with a deviation from the optimal value not more than a fraction of a percent. Here, it is also shown the geometrical parameter $p$. It is clearly to be seen, also as it was earlier (Fig. 15), that the lowest $p$ corresponds to the maximal radius of curvature at the equator. However, 

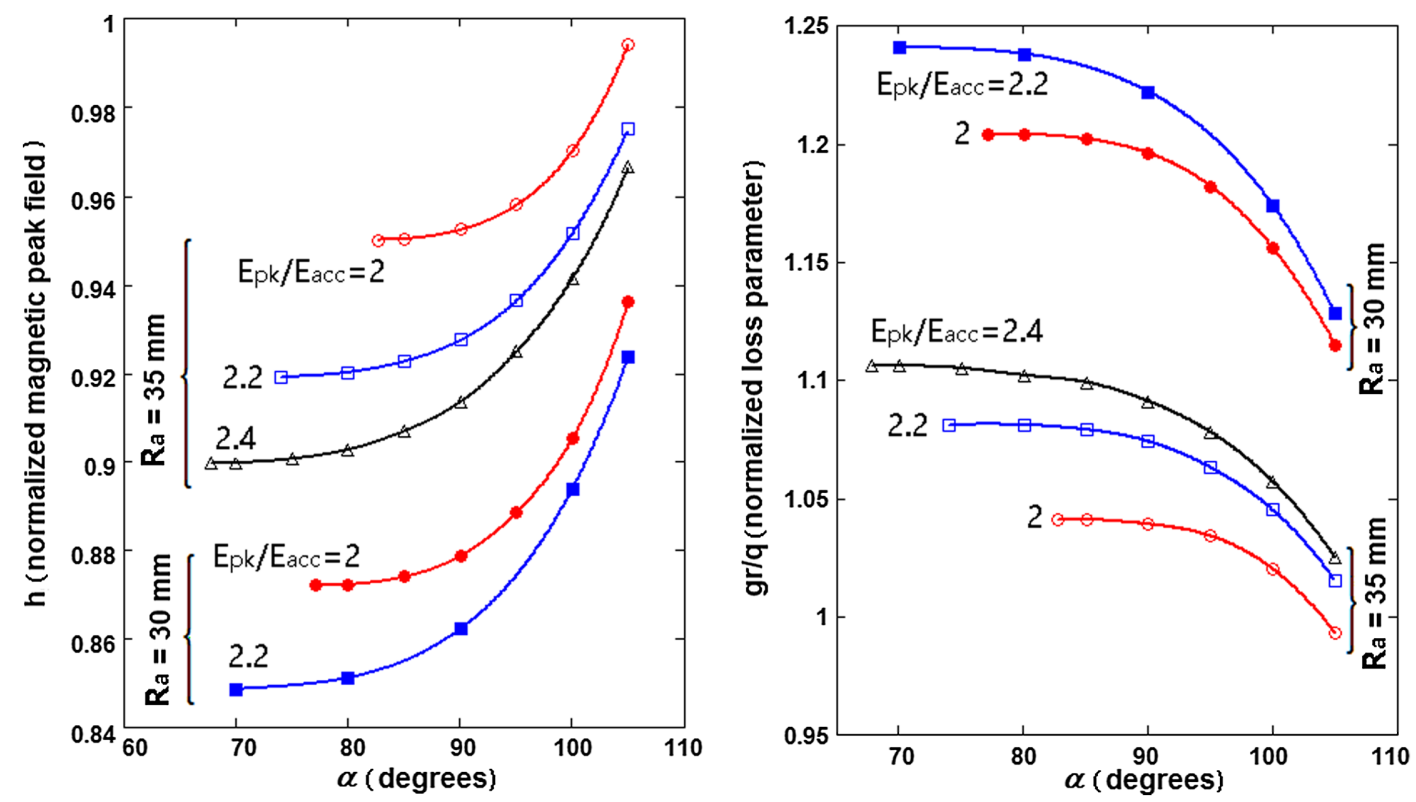

FIG. 17. Normalized magnetic peak field and normalized loss parameter for different angles of the wall slope for $\beta=1$.

making the equator flat can lead to the one-point multipactor.

\section{E. Elliptic cavities with $\beta=0.6$}

Results of optimization for $\beta=0.6$ are presented in Fig. 22 and in Table VI. Here, for comparison, data from [16] are also shown for the SNS cavity with $\beta=0.61$. After scaling from $805 \mathrm{MHz}$ to $1300 \mathrm{MHz}$ the aperture radius $R_{a}$
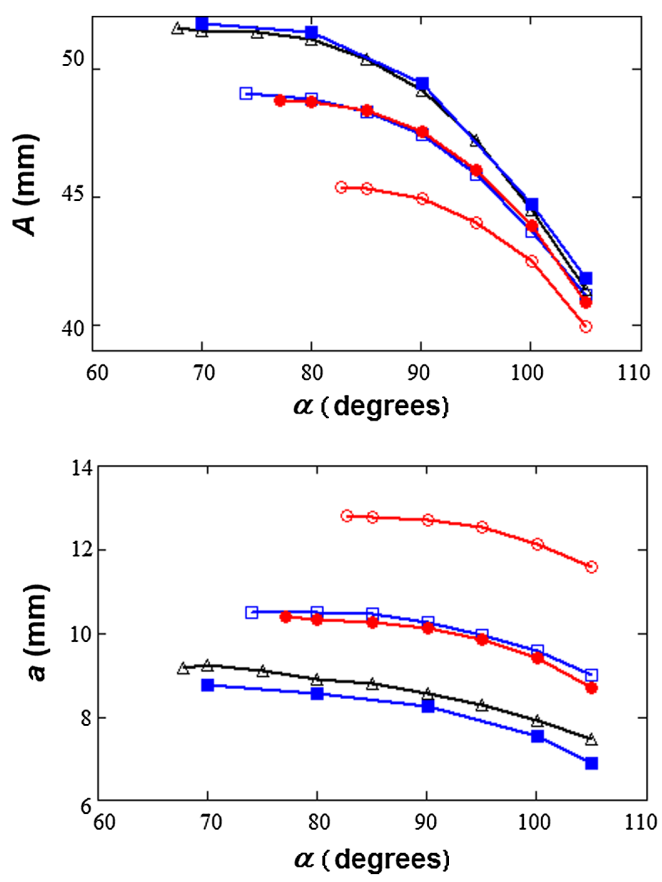

changed from 43 to $26.627 \mathrm{~mm}$. Point $a$ for the normalized magnetic field is taken from this paper: $B_{\text {peak }} / E_{\text {acc }}=$ $5.73 \mathrm{mT} /(\mathrm{MV} / \mathrm{m})$ that corresponds to $h=1.364$. Our calculations, however, give for this point $h=1.294$ (point $b$ ), and after optimization this shape was slightly improved-to $h=1.277$ (point $c$ ).

The values of $R_{a}$ and $E_{\mathrm{pk}} / E_{\mathrm{acc}}$ are chosen so that the data for the SNS cavity are between these values, and the difference in $\beta$ is not large.
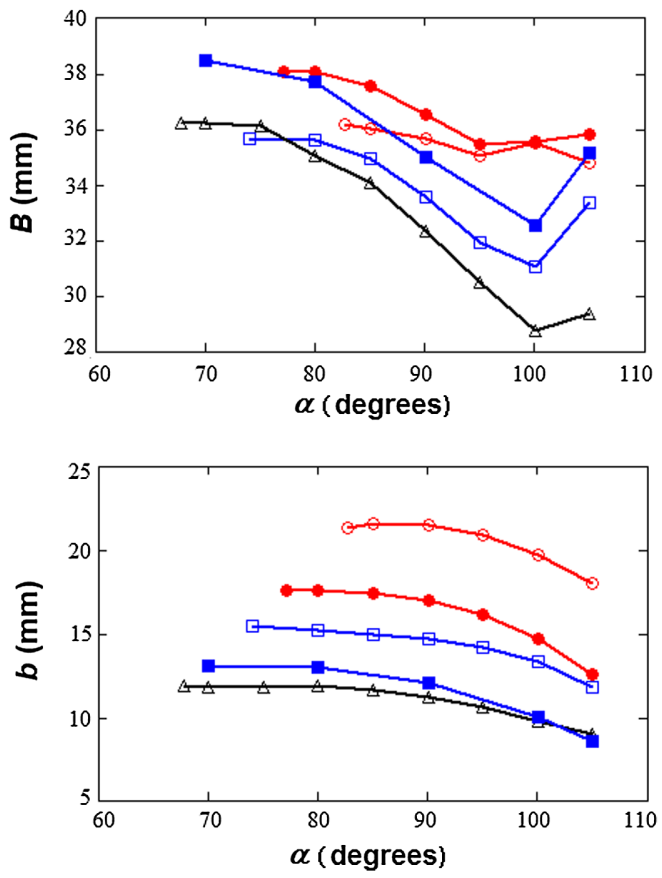

FIG. 18. Elliptic half-axes for the previous figure. Designation of points are the same. See details in Sec. X A. 


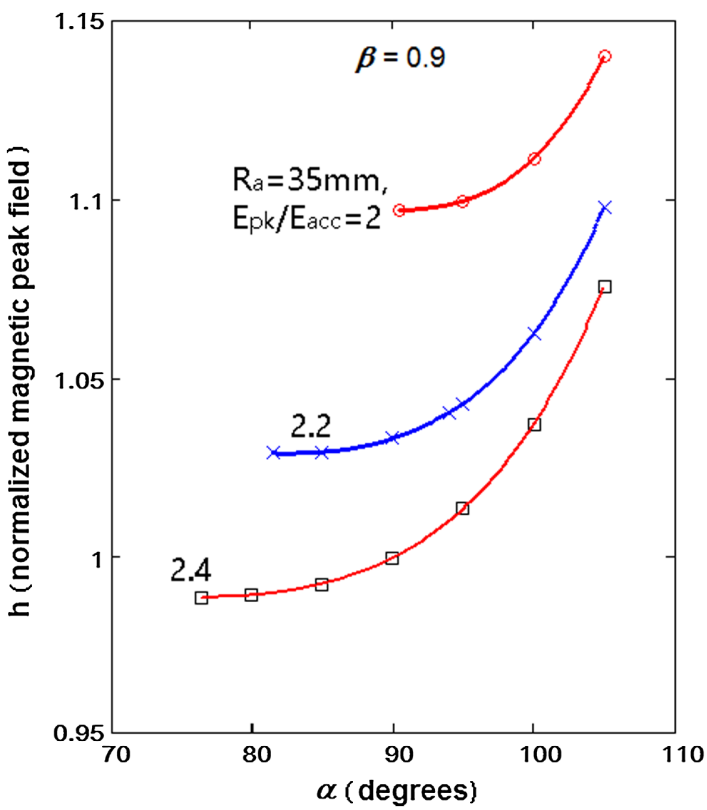

FIG. 19. Normalized magnetic peak field for different angles of the wall slope for $\beta=0.9$.

TABLE III. Values of $h=H_{\mathrm{pk}} / E_{\mathrm{acc}} / 42$, and dimensions of optimized inner cell (two elliptic arcs and a straight segment) vs the slope angle $\alpha$. Optimization for $\min H_{\mathrm{pk}} / E_{\mathrm{acc}}$ (or $\min h$ ).

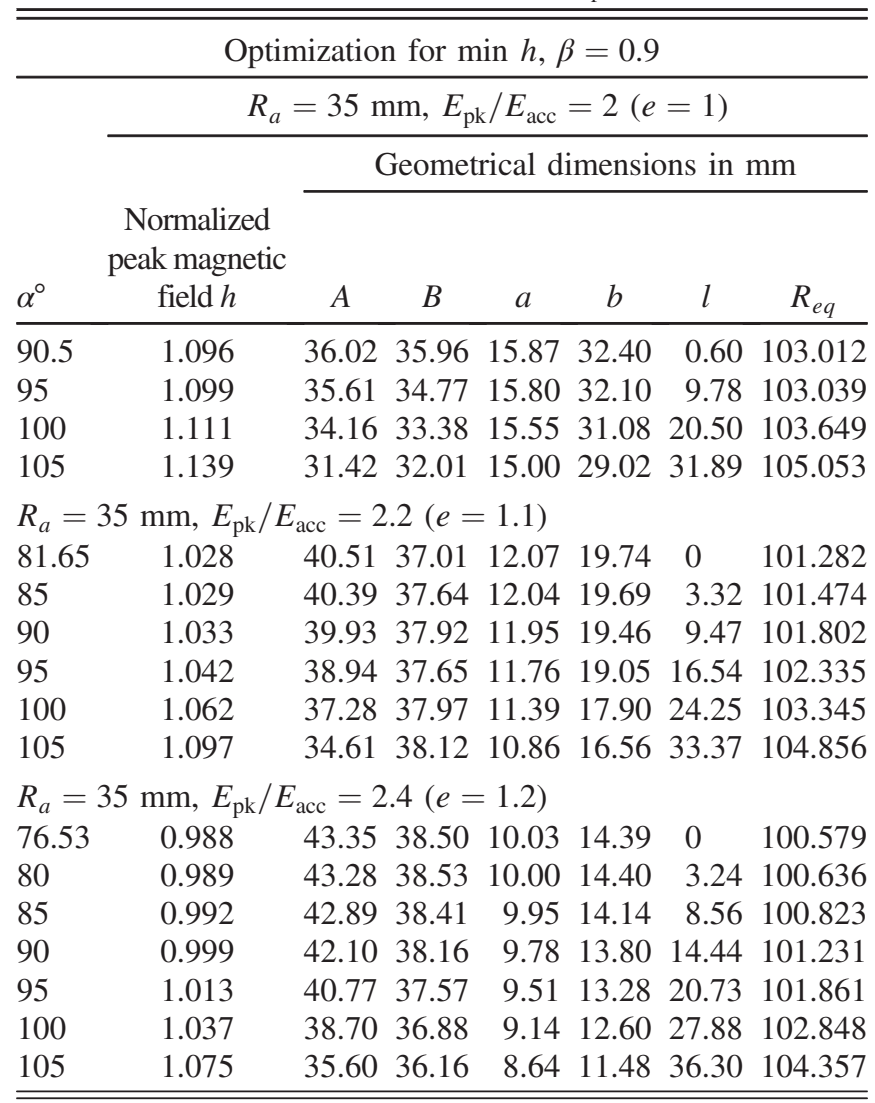

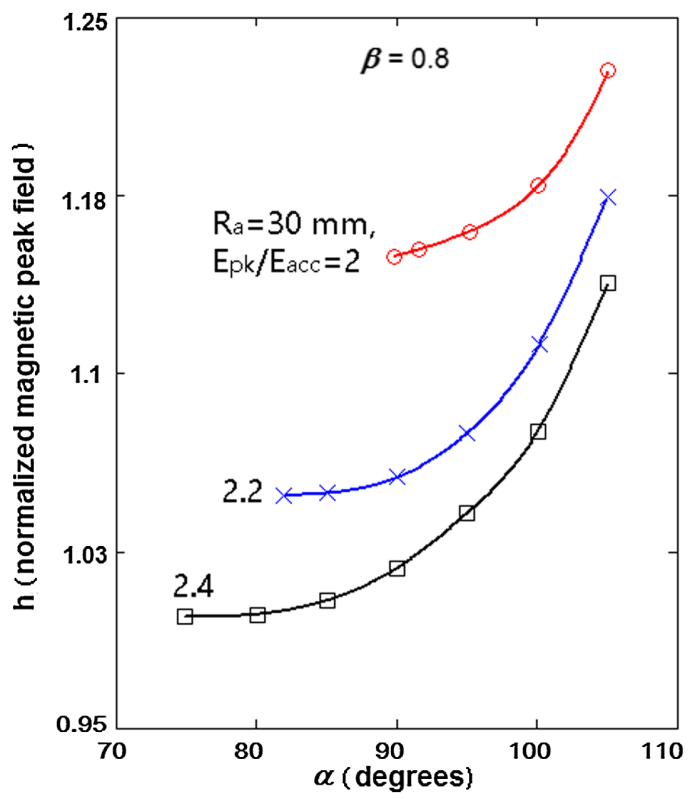

FIG. 20. Normalized magnetic peak field for different angles of the wall slope for $\beta=0.8$.

TABLE IV. Values of $h=H_{\mathrm{pk}} / E_{\mathrm{acc}} / 42$, and dimensions of optimized inner cell (two elliptic arcs and a straight segment) vs the slope angle $\alpha$. Optimization for $\min H_{\mathrm{pk}} / E_{\mathrm{acc}}$ (or $\min h$ ).

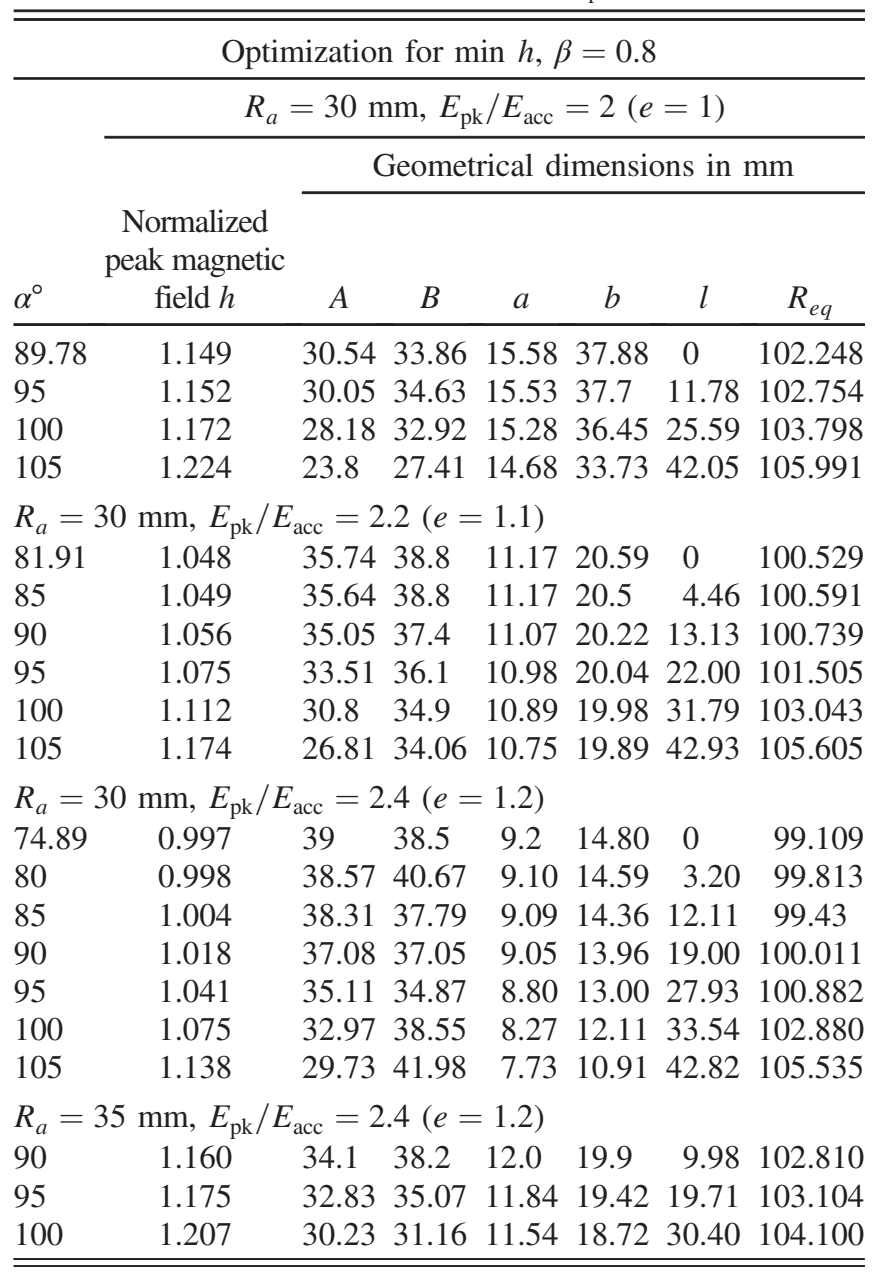




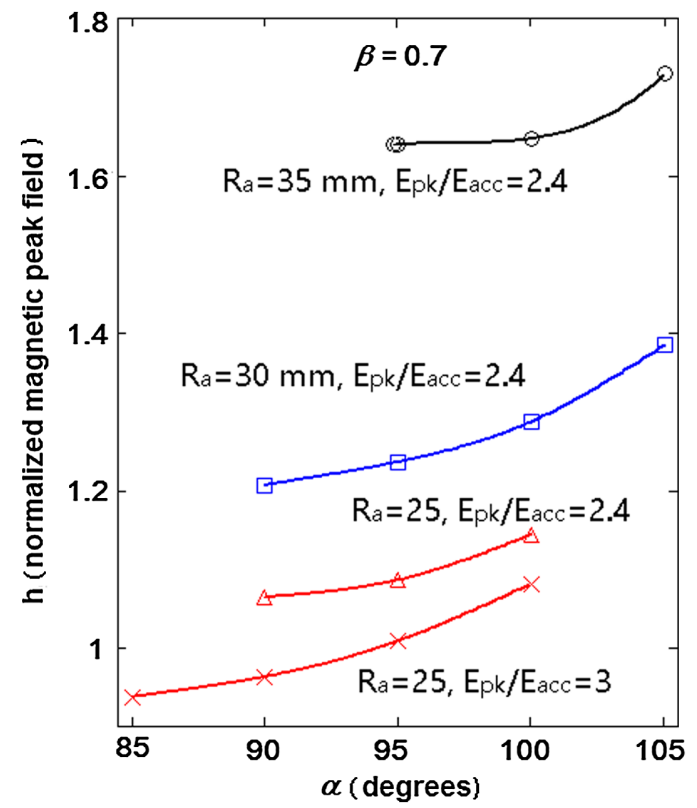

FIG. 21. Normalized magnetic peak field for different angles of the wall slope for $\beta=0.7$.

\section{F. Elliptic cavities with $\beta=0.5$}

Results of optimization for $\beta=0.5$ are presented in Fig. 23 and in Table VII. Here are also shown for

TABLE V. Values of $h=H_{\mathrm{pk}} / E_{\mathrm{acc}} / 42$, and dimensions of optimized inner cell (two elliptic arcs and a straight segment) vs the slope angle $\alpha$. Optimization for $\min H_{\mathrm{pk}} / E_{\mathrm{acc}}$ (or $\min h$ ).

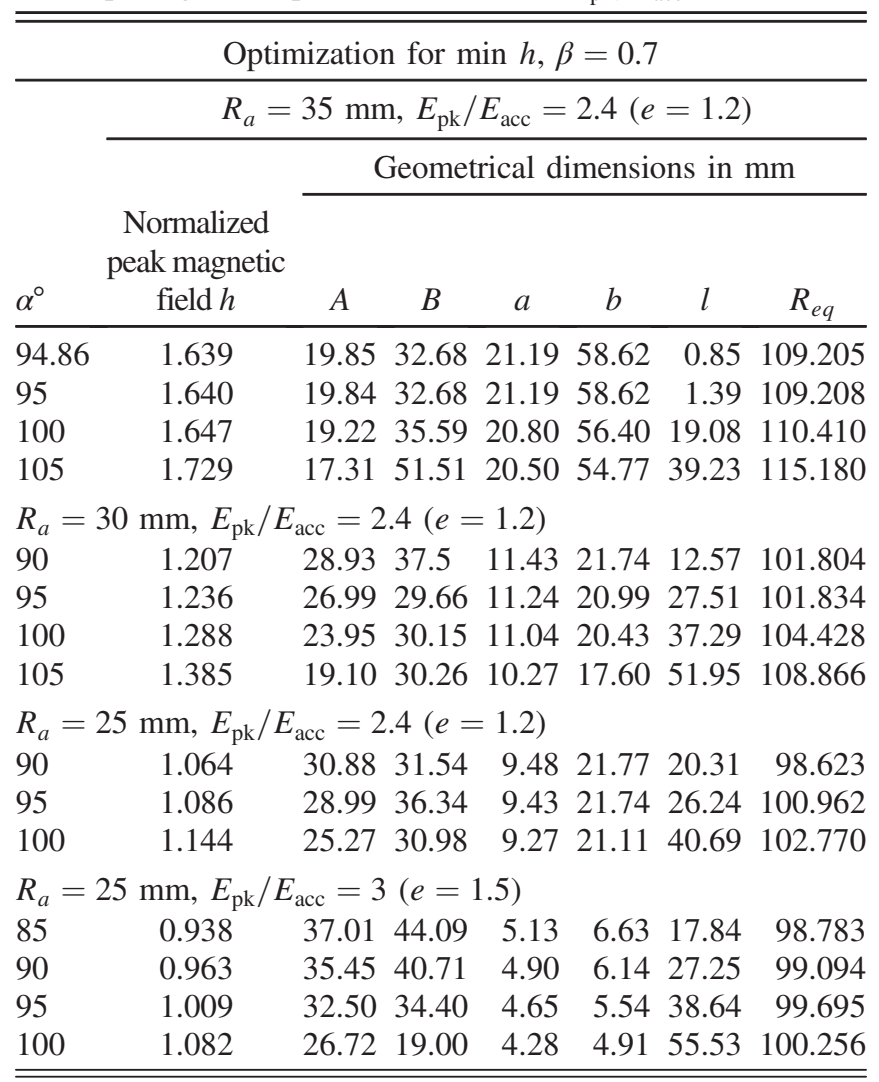

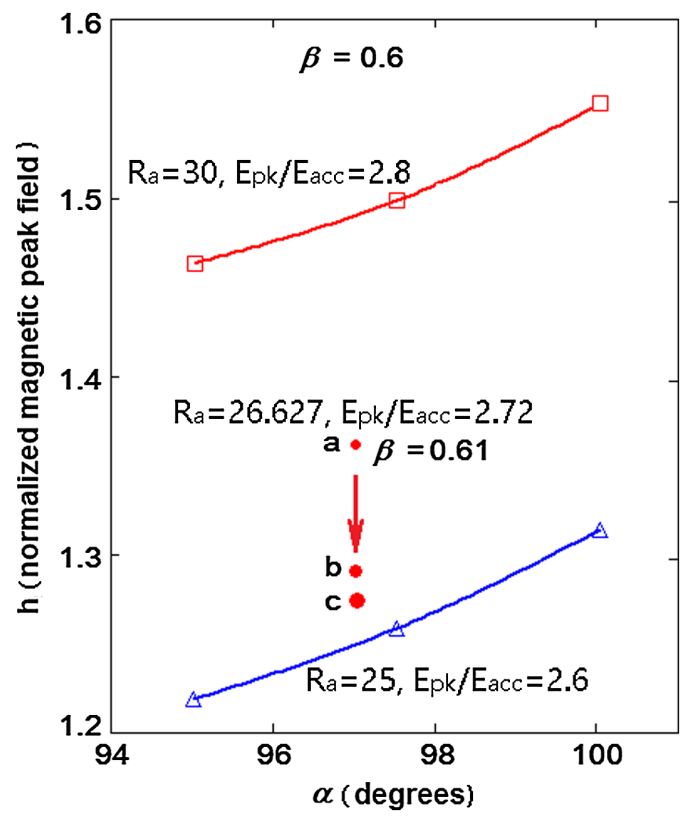

FIG. 22. Normalized magnetic peak field for different angles of the wall slope for $\beta=0.6$. Points $\mathrm{a}, \mathrm{b}$, and $\mathrm{c}$ are shown for comparison with data from [16], see text.

comparison data from [16] for the TRASCO-ASH cavity, for $704.4 \mathrm{MHz}$ (on the left), and RIA cavity, for $805 \mathrm{MHz}$ (on the right), both with $\beta=0.47$. After scaling to $1300 \mathrm{MHz}$ their aperture radii $R_{a}$ changed to $21.674 \mathrm{~mm}$ and $23.902 \mathrm{~mm}$, respectively. Points $a$ and $d$ for the normalized magnetic field are taken from this paper. Our calculations, however, give for these points somewhat lower values (points $b$ and $e$ ), and after optimization this shape was even slightly more improved (point $c$ and $f$ ).

The values of $R_{a}$ and $E_{\mathrm{pk}} / E_{\mathrm{acc}}$ for the curves are chosen so that the data for the TRASCO-ASH and RIA cavity are

TABLE VI. Values of $h=H_{\mathrm{pk}} / E_{\mathrm{acc}} / 42$, and dimensions of optimized inner cell (two elliptic arcs and a straight segment) vs the slope angle $\alpha$. Optimization for $\min H_{\mathrm{pk}} / E_{\mathrm{acc}}$ (or $\min h$ ).

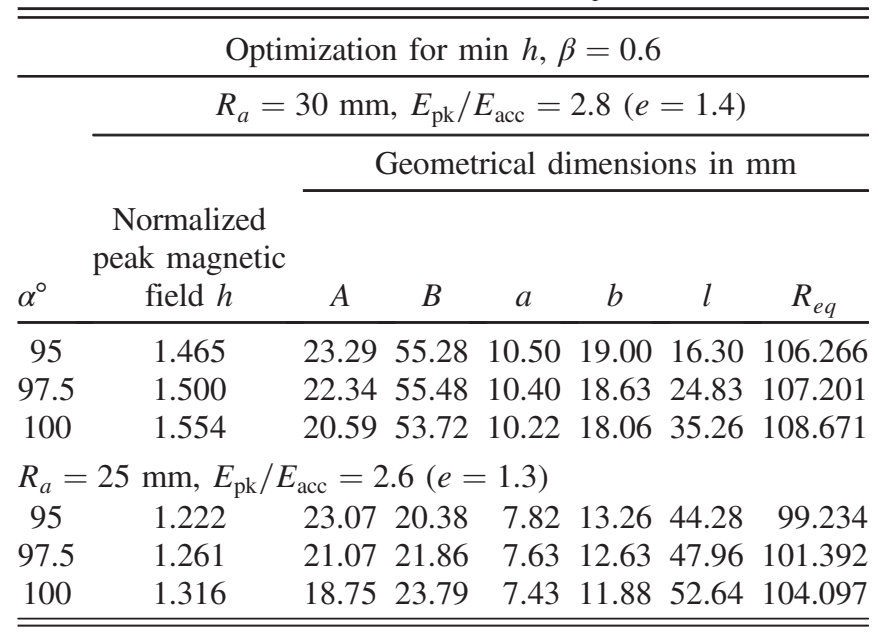




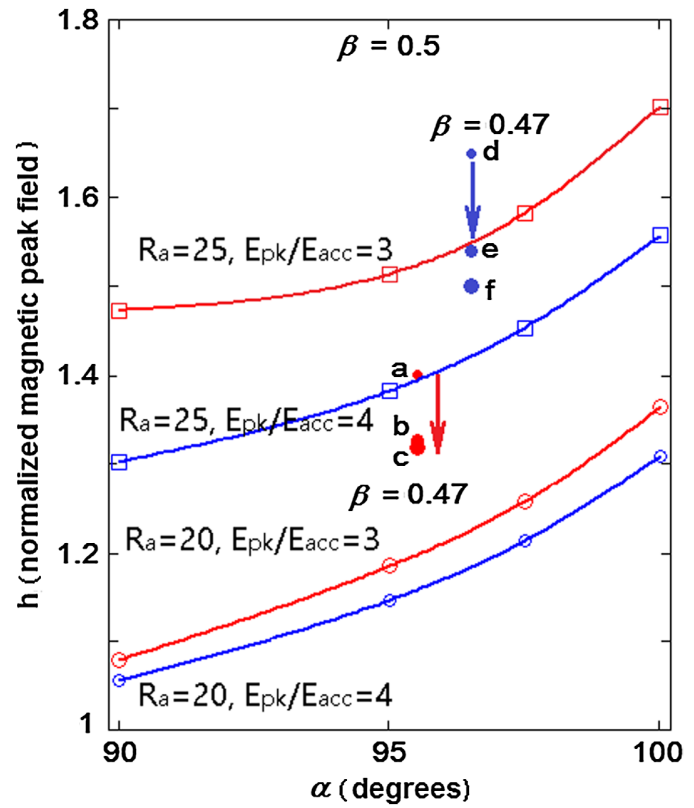

FIG. 23. Normalized magnetic peak field for different angles of the wall slope for $\beta=0.5$. Points $\mathrm{a}, \mathrm{b}$, and $\mathrm{c}$, also as $\mathrm{d}, \mathrm{e}, \mathrm{f}$ are shown for comparison with data from [16], see text.

TABLE VII. Values of $h=H_{\mathrm{pk}} / E_{\mathrm{acc}} / 42$, and dimensions of optimized inner cell (two elliptic arcs and a straight segment) vs the slope angle $\alpha$. Optimization for $\min H_{\mathrm{pk}} / E_{\mathrm{acc}}$ (or $\min h$ ).

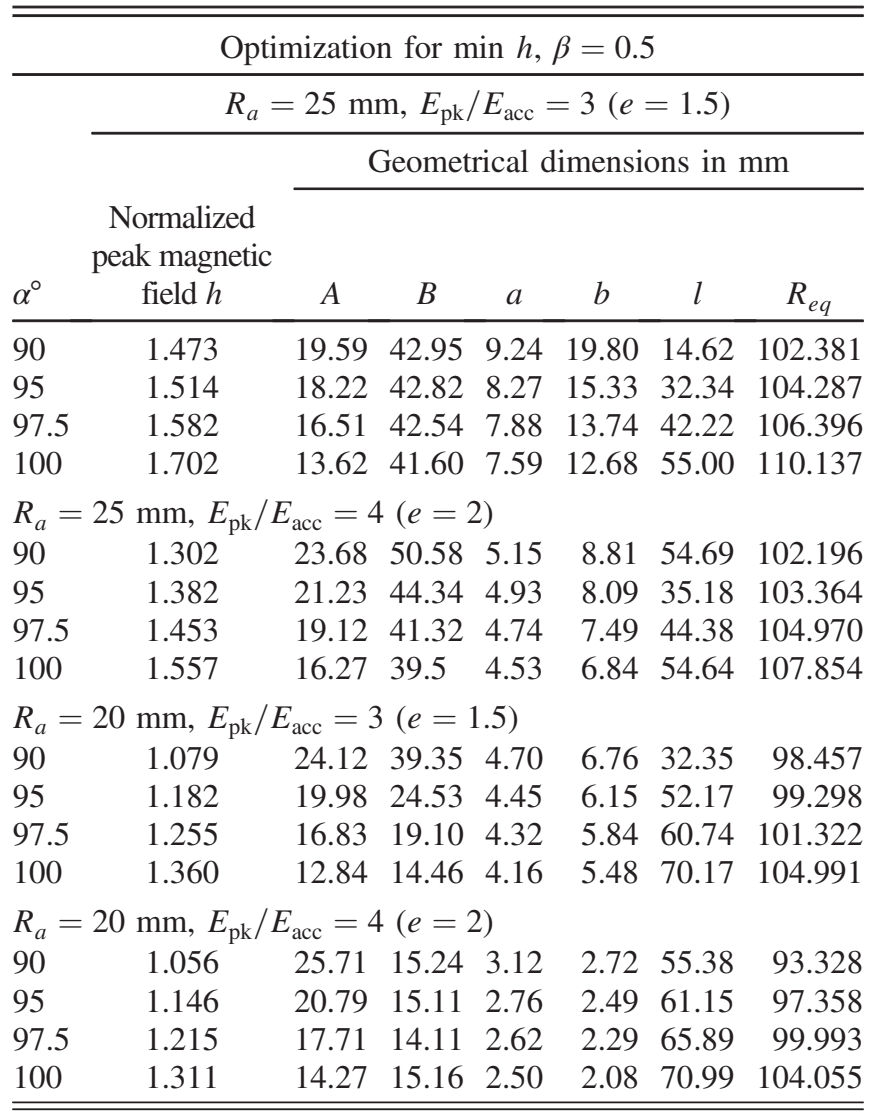

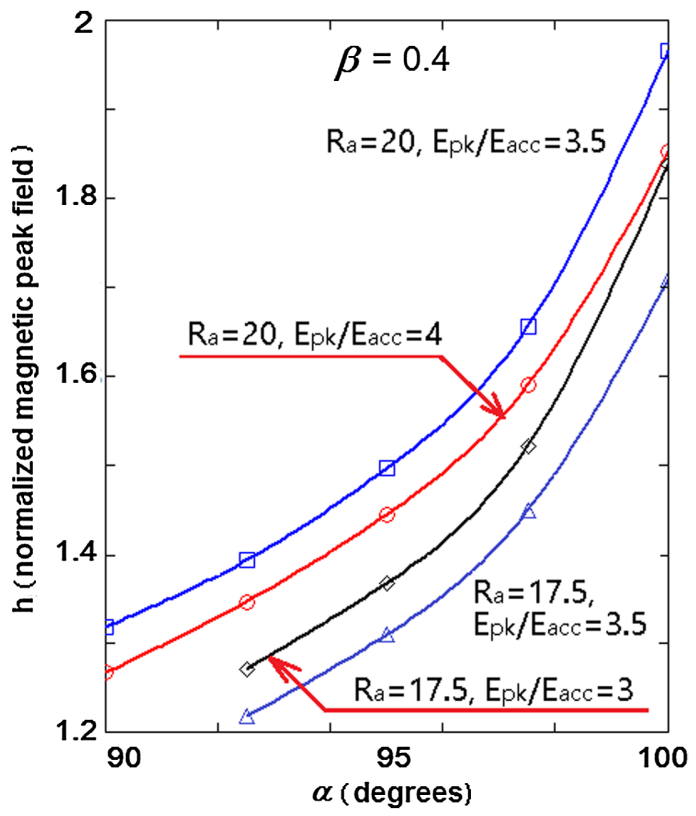

FIG. 24. Normalized magnetic peak field for different angles of the wall slope for $\beta=0.4$.

TABLE VIII. Values of $h=H_{\mathrm{pk}} / E_{\mathrm{acc}} / 42$, and dimensions of optimized inner cell (two elliptic arcs and a straight segment) vs the slope angle $\alpha$. Optimization for $\min H_{\mathrm{pk}} / E_{\mathrm{acc}}($ or $\min h$ ) and $r_{c} \geq 3 \mathrm{~mm}$.

\begin{tabular}{|c|c|c|c|c|c|c|c|}
\hline \multicolumn{8}{|c|}{ Optimization for $\min h, \beta=0.4$} \\
\hline \multirow[b]{3}{*}{$\alpha^{\circ}$} & \multicolumn{7}{|c|}{$R_{a}=20 \mathrm{~mm}, E_{\mathrm{pk}} / E_{\mathrm{acc}}=3.5(e=1.75)$} \\
\hline & \multirow{2}{*}{$\begin{array}{l}\text { Normalized } \\
\text { peak magnetic } \\
\text { field } h\end{array}$} & \multicolumn{6}{|c|}{ Geometrical dimensions in $\mathrm{mm}$} \\
\hline & & $A$ & $B$ & $a$ & $b$ & $l$ & $R_{e q}$ \\
\hline 90 & 1.318 & 18.34 & 30.00 & 4.72 & 6.99 & 40.21 & 97.197 \\
\hline 92.5 & 1.393 & 16.36 & 25.14 & 4.61 & 6.64 & 48.88 & 98.508 \\
\hline 95 & 1.497 & 13.42 & 16.76 & 4.51 & 6.37 & 60.14 & 100.435 \\
\hline 97.5 & 1.655 & 9.42 & 9.89 & 4.41 & 6.07 & 71.94 & 104.848 \\
\hline 100 & 1.965 & 2.86 & 4.14 & 4.28 & 5.78 & 92.89 & 119.033 \\
\hline \multicolumn{8}{|c|}{$R_{a}=20 \mathrm{~mm}, E_{\mathrm{pk}} / E_{\mathrm{acc}}=4(e=2)$} \\
\hline 90 & 1.268 & 19.43 & 34.86 & 3.63 & 4.39 & 38.70 & 97.952 \\
\hline 92.5 & 1.348 & 17.18 & 20.06 & 3.54 & 4.20 & 54.33 & 97.310 \\
\hline 95 & 1.447 & 14.03 & 12.14 & 3.46 & 3.98 & 64.62 & 99.180 \\
\hline 97.5 & 1.595 & 9.89 & 5.78 & 3.4 & 3.76 & 75.35 & 103.261 \\
\hline 100 & 1.860 & 4.80 & 7.55 & 3.25 & 3.52 & 87.79 & 114.847 \\
\hline \multicolumn{8}{|c|}{$R_{a}=17.5 \mathrm{~mm}, E_{\mathrm{pk}} / E_{\mathrm{acc}}=3(e=1.5)$} \\
\hline 92.5 & 1.272 & 15.45 & 3.98 & 4.76 & 7.56 & 65.47 & 93.882 \\
\hline 95 & 1.369 & 12.44 & 5.57 & 4.64 & 7.16 & 69.23 & 98.025 \\
\hline 97.5 & 1.522 & 8.6 & 4.84 & 4.5 & 6.72 & 77.13 & 103.869 \\
\hline 100 & 1.841 & 1.98 & 7.96 & 4.35 & 6.3 & 99.26 & 123.349 \\
\hline \multicolumn{8}{|c|}{$R_{a}=17.5 \mathrm{~mm}, E_{\mathrm{pk}} / E_{\mathrm{acc}}=3.5(e=1.75)$} \\
\hline 92.5 & 1.219 & 16.65 & 6.29 & 3.52 & 4.13 & 66.43 & 93.979 \\
\hline 95 & 1.313 & 13.46 & 5.57 & 3.41 & 3.88 & 71.33 & 97.420 \\
\hline 97.5 & 1.454 & 9.89 & 7.71 & 3.29 & 3.61 & 76.33 & 103.192 \\
\hline 100 & 1.721 & 4.67 & 11.54 & 3.16 & 3.33 & 90.29 & 116.067 \\
\hline
\end{tabular}


between these values, and the difference in $\beta$ is not large. Values of surface overvoltage are also in between the parameters used for the curves: $E_{\mathrm{pk}} / E_{\mathrm{acc}}=3.57$ for the TRASCO-ASH and 3.41 for the RIA cavity. One can see that the shapes are very close to optimal ones even in spite of the chosen ratio $R=1$ for the RIA cavity (on the right). For the TRASCO-ASH cavity this ratio is 1.6 and the presented shape is practically optimal.

\section{G. Elliptic cavities with $\beta=0.4$}

Results of optimization for $\beta=0.4$ are presented in Fig. 24 and in Table VIII.

Small values of the elliptic half axes for $\beta=0.4$ lead to small curvature radius at $f=1300 \mathrm{MHz}$ which as previously explained poses difficulties in fabrication of the cavity. This suggests to consider elliptic cavities for low $\beta$ applications only at lower frequencies.

\section{CONCLUSION}

Tables and graphs for dependencies of the minimal values of $H_{\mathrm{pk}} / E_{\mathrm{acc}}$ on the cavity wall slope angle are presented for different values of $\beta$ from 1 to 0.4 with a step of 0.1 , different aperture radii, and peak surface electric fields. Geometrical data of the cavities are included in the tables. These data can be used for a preliminary design of elliptic SRF cavities for different accelerating structures. The data are given for the frequency of $1300 \mathrm{MHz}$ but they are valid for a wide range of frequencies because most of the important parameters and figures of merit of the SRF cavities, depend on the cavity shape only, not on the size.

A new property of the elliptic cavity shape is revealed: it is possible to maintain the minimal $H_{\mathrm{pk}} / E_{\mathrm{acc}}$ but to change the shape of the cavity. This makes it possible to avoid multipactor keeping the optimal shape. Examples of this solution are presented. This change of the shape can be also used for detuning the most adverse higher order modes or/ and for decreasing the Lorentz force detuning.

Geometrical constraints for the shape of elliptic cavities are analyzed. Small values of the elliptic half-axes for the cavities with smallest $\beta$ suggest to conclude that such cavities can be fabricated for the frequencies at about two times lower than the "most popular" frequency of $1300 \mathrm{MHz}$. As practice shows, this is true for the existing examples of elliptic cavities. We can confirm the earlier statement that the value of $\beta=0.4$ should be the lowest limit for this class of cavities.

Comparison of the presented optimal shapes with some existing cavities is done and a reasonable agreement in their geometry is demonstrated.

\section{ACKNOWLEDGMENTS}

This research was supported by Deutsche Forschungsgemeinschaft (DFG) Grant No. RI 814/25-1, Bundesministerium für Bildung und Forschung (BMBF) Grant No. 05K13HR1 and Future Circular Collider (FCC) study at CERN.

[1] J. R. Delayen, in Proceedings of the 10th Workshop on RF superconductivity, Tsukuba, edited by S. Noguchi (KEK, Tsukuba, 2001), pp. 152-161, http://accelconf.web.cern .ch/accelconf/srf01/papers/fa007.pdf.

[2] A. Facco, in Proceedings of the 12th Workshop on RF superconductivity, Ithaca, New York, edited by S. Belomestnykh, M. Liepe, and H. Padamsee (Cornell University, Ithaca, New York, 2001), pp. 21-33, http://www.lns .cornell.edu/public/SRF2005/pdfs/SuA04.pdf.

[3] P. Pierini, D. Barni, A. Bosotti, G. Ciovati, and C. Pagani, in Proceedings of the 9th Workshop on RF superconductivity, La Fonda Hotel, Santa Fe, New Mexico (1999), pp. 380-383, https://accelconf.web.cern.ch/accelconf/ SRF99/papers/wep004.pdf.

[4] B. Aune et al., Superconducting TESLA cavities, Phys. Rev. ST Accel. Beams 3, 092001 (2000).

[5] J. Sekutowicz, P. Kneisel, G. Ciovati, and H. Wang, Report No. JLab-TN-02-023, 2002.

[6] V. Shemelin, H. Padamsee, and R. L. Geng, Optimal cells for TESLA accelerating structure, Nucl. Instrum. Methods Phys. Res., Sect. A 496, 1 (2003).

[7] B. Riemann, T. Weis, and A. Neumann, in Proceedings of the ICAP 2012 Conference, Rostock-Warnemünde (University of Rostock, Rostock, 2012), pp. 167-169, http://accelconf .web.cern.ch/accelconf/icap2012/papers/wep14.pdf.

[8] E. Haebel, A. Mosnier, and J. Sekutowicz, in Proceedings of the XV International Conference on HEACC, Hamburg 1992, Vol. 2.

[9] D. A. Edwards, TESLA Test Facility Linac Design Report No. TESLA 95-01 DESY Print, 1995.

[10] D. G. Myakishev and V. P. Yakovlev, in Proceedings of the Particle Accelerator Conference, Dallas, TX, 1995 (IEEE, New York, 1995).

[11] http://www.euclidtechlabs.com/SLANS/slans.php.

[12] S. Belomestnykh, Cornell University LEPP Report No. SRF 941208-13, 1994.

[13] V. Shemelin, Cornell University LEPP Report No. SRF 070614-02, 2007.

[14] J. J. Moré, Numerical Analysis (Springer, New York, 1978), p. 105.

[15] W. R. Gilks, Markov Chain Monte Carlo (John Wiley \& Sons, New York, 2005).

[16] C. Pagani, D. Barni, A. Bosotti, P. Pierini, and G. Ciovatti, in Proceedings of the 10th Workshop on RF Superconductivity (KEK, Tsukuba, 2001), pp. 115-122.

[17] V. Shemelin, Multipactor in crossed rf fields on the cavity equator, Phys. Rev. ST Accel. Beams 16, 012002 (2013). 